NBER WORKING PAPER SERIES

\title{
FUTURES PRICES IN A PRODUCTION ECONOMY WITH INVESTMENT CONSTRAINTS
}

\author{
Leonid Kogan \\ Dmitry Livdan \\ Amir Yaron \\ Working Paper 11509 \\ http://www.nber.org/papers/w11509
NATIONAL BUREAU OF ECONOMIC RESEARCH
1050 Massachusetts Avenue
Cambridge, MA 02138
June 2005

We are grateful to Kerry Back, Pierre Collin-Dufresne, Francis Longstaff and Craig Pirrong, as well as seminar participants at Northwestern University, Texas A\&M University, 2004 Western Finance Association meeting, 2004 Society of Economic Dynamics meeting, and 2004 European Econometric Society meeting for useful suggestions. We also thank Krishna Ramaswamy for providing us with the futures data. Financial support from the Rodney L. White center for Financial Research at the Wharton School is gratefully acknowledged. Kogan: Phone (617) 253-2289, Fax (617) 258-6855. Livdan: Phone (713) 743-4813, Fax (713) 743-4755. Yaron: Phone (215) 898-1241, Fax: (215) 898-6200. The views expressed herein are those of the author(s) and do not necessarily reflect the views of the National Bureau of Economic Research.

(C2005 by Leonid Kogan, Dmitry Livdan and Amir Yaron. All rights reserved. Short sections of text, not to exceed two paragraphs, may be quoted without explicit permission provided that full credit, including () notice, is given to the source. 
Futures Prices in a Production Economy with Investment Constraints

Leonid Kogan, Dmitry Livdan and Amir Yaron

NBER Working Paper No. 11509

July 2005

JEL No. G12, G13

\section{$\underline{\text { ABSTRACT }}$}

We document a new stylized fact regarding the term-structure of futures volatility. We show that the relation between the volatility of futures prices and the slope of the term structure of prices is non-monotone and has a "V-shape". This aspect of the data cannot be generated by basic models that emphasize storage while this fact is consistent with models that emphasize investment constraints or, more generally, time-varying supply-elasticity. We develop an equilibrium model in which futures prices are determined endogenously in a production economy in which investment is both irreversible and is capacity constrained. Investment constraints affect firms' investment decisions, which in turn determine the dynamic properties of their output and consequently imply that the supply-elasticity of the commodity changes over time. Since demand shocks must be absorbed either by changes in prices, or by changes in supply, time-varying supply-elasticity results in time-varying volatility of futures prices. Calibrating this model, we show it is quantitatively consistent with the aforementioned "V-shape" relation between the volatility of futures prices and the slope of the term-structure.

$\begin{array}{lll}\text { Leonid Kogan } & \text { Dmitry Livdan } & \text { Amir Yaron } \\ \text { Sloan School of Management, } & \text { C.T. Bauer School of Business } & \text { The Wharton School } \\ \text { MIT } & \text { University of Houston } & \text { University of Pennsylvania } \\ 50 \text { Memorial Drive, E52-434 } & \text { 220F Melcher Hall } & \text { 2256 Steinberg-Dietrich Hall } \\ \text { Boston, MA 02142 } & \text { Houston, TX 77204 } & \text { Philadelphia, PA 19104-6367 } \\ \text { and NBER } & \text { dlivdan@ uh.edu } & \text { and NBER } \\ \text { lkogan@mit.edu } & & \text { yaron@wharton.upenn.edu }\end{array}$




\section{Introduction}

In recent years commodity markets have experienced dramatic growth in trading volume, the variety of contracts, and the range of underlying commodities. There also has been a great demand for derivative instruments utilizing operational contingencies embedded in delivery contracts. For all these reasons there is a widespread interest in models for pricing and hedging commodity-linked contingent claims. Besides practical interest, commodities offer a rich variety of empirical properties, which make them strikingly different from stocks, bonds and other conventional financial assets. Notable properties of futures include, among others: (i) Commodity futures prices are often "backwardated" in that they decline with time-to-delivery, (ii) Spot and futures prices are mean reverting for many commodities, (iii) Commodity prices are strongly heteroscedastic and price volatility is correlated with the degree of backwardation, and (iv) Unlike financial assets, many commodities have pronounced seasonalities in both price levels and volatilities.

The 'theory of storage' of Kaldor (1939), Working (1948, 1949) and Telser (1958) has been the foundation of the theoretical explorations of futures/forward prices and convenience yields (value of the immediate ownership of the physical commodity). Based on this theory researchers have adopted two approaches to modelling commodity prices. The first approach is mainly statistical in nature and requires an exogenous specification of the 'convenience yield' process for a commodity (e.g., Brennan and Schwartz (1985), Brennan (1991), and Schwartz (1997)). The second strand of the literature derives the price processes endogenously in an equilibrium valuation framework with competitive storage (e.g., Williams and Wright (1991), Deaton and Laroque (1992, 1996), Routledge, Seppi, and Spatt (2000)). The appealing aspect of this approach is its ability to link the futures prices to the level of inventories and hence derive additional testable restrictions on the price processes.

From a theoretical perspective the models based on competitive storage ignore the 
production side of the economy, and consequently they suffer from an important limitation. Inventory dynamics have little if any impact on the long-run properties of commodity prices, which in such models are driven mostly by the exogenously specified demand process. In particular, prices in such models tend to mean revert too fast relative to what is observed in the data (see Routledge et. al. (2000)), and more importantly these models can not address the rich term-structure dynamics of return volatility.

In this paper we document an important new stylized fact regarding the property of the term structure of volatility of futures prices. We demonstrate that the relation between the volatility of futures prices and the slope of the forward curve (the basis) is non-monotone and has a "V-shape". Specifically, conditional on a negatively sloped term structure, the relation between the volatility of futures prices and the slope of the forward curve is negative. On the other hand, conditional on a positively sloped term structure, the relation between the volatility and the basis is positive. This aspect of the data cannot be generated by basic models that emphasize storage, since such models imply a monotone relation between futures price volatility and the slope of the forward curve (see Routledge et. al. (2000)).

In light of the aforementioned stylized fact, we explore an alternative model characterizing the mechanism of futures price formation. Future prices are determined endogenously in an equilibrium production economy featuring constraints on investment, such as irreversibility. Investment constraints affect firms investment decisions, which in turn determine the dynamic properties of their output. In particular, because of the binding constraints on investment, supply-elasticity of the commodity changes over time. Since demand shocks must be absorbed either by changes in prices, or by changes in supply, time-varying supplyelasticity results in time-varying volatility of futures prices. In our calibration below we show that the model can also generate these patterns in a manner that is quantitatively similar to the data. 
There exists very little theoretical work investigating the pricing of futures on commodities using production economy framework. Casassus, Collin-Dufresne and Routledge (2004) also analyze spot and futures oil prices in a general equilibrium production economy but with fixed investment costs and two goods. While also a production economy, the structure of their model is different and leads to a regime-switching model of futures prices. This regime-switching feature of the model generates the key implication - a nonunique relation between the level of spot prices and the convenience yield, or the state of the economy. In comparison, our model assumes a relatively standard production and investment technology and provides parsimonious and intuitive explanation of the new stylized fact we uncover - a "V-shaped" relation between the slope of the forward curve and the conditional volatility of futures prices.

The rest of the paper is organized as follows. In Section 2 we describe our data analysis regarding future prices. Section 3 develops the model's economy as well as presents the spot price and prices of financial assets derived in a competitive equilibrium setting. In Section 4, we study quantitative implications of the model. Section 5 provides conclusions.

\section{Empirical Analysis}

We concentrate our empirical study on three commodities: crude oil, heating oil, and unleaded gasoline. Our data consists of daily futures prices for three contracts: NYMEX heating oil contract (HO) for the period from 1979 to 2000, NYMEX light sweet crude oil contract (CL) for the period from 1982 to 2000, and NYMEX unleaded New York harbor gasoline futures (HU) for the period from 1985 to 2000. Following previous work by Routledge et. al (2000), the data is sorted by contract horizon with the 'one-month' contract being the contract with the earliest delivery date, the 'two-month' contract having next earliest 
delivery date, etc. ${ }^{1}$. We consider contracts with up to 12 months to delivery when since liquidity and data availability is good for these horizons for all three contracts used in this study. $^{2}$ Since we are using daily data, our dataset is sufficiently large: it ranges from 2500 to 3500 data points across different contracts and maturities.

Instead of directly using futures prices, $P(t, T)$, we use daily percent changes, $R(t, T)=$ $\frac{P(t, T)}{P(t-1, T)}$. Percent price changes are not susceptible as much as price levels to seasonalities and trends, and therefore their volatility is more suitable for empirical analysis. We then proceed by constructing the term structure of the unconditional and conditional volatilities of daily percent changes on futures for all three contracts. In calculating conditional moments we classify the futures curve at each date $t$ as to whether the curve was in backwardation or contango the previous trading day (based on the shortest and third shortest maturity prices at that time). Figures $1-3$ show these conditional and unconditional daily volatilities for futures percent changes for CL, HO, and HU contracts respectively. The behavior of crude oil (CL) contracts was studied by Routledge et. al. (2000). We find, as they did, that the volatility of futures prices is higher when the forward curve is in backwardation. This has been interpreted as evidence in favor of the standard storage theories, emphasizing the effect of inventory stock-outs on price volatility. Note, however, that the pattern is reversed for heating oil (HO) futures. There is no discernable relation for gasoline (HU) contracts. These basic observations suggests that evidence in favor of the standard storage theory is, at best, mixed.

\footnotetext{
${ }^{1}$ In our data set, for each of the commodities, on any given calendar day there are several contracts available with different time to delivery measured in days. The difference in delivery times between these contracts is at least 32 days or more. We utilize the following procedure for converting delivery times to the monthly scale. For each contract we divide the number of days it has left to maturity by 30 (the average number of days in a month), and then round off the resultant. For days when contract with time to delivery of less than 15 days is traded, we add one "month" to the contract horizon obtained using the above procedure for all contracts traded on such days. The data is then sorted into bins based on the contract horizon measured in months.

${ }^{2}$ We refer to this time to delivery as time to maturity throughout the paper.
} 
Next, we study the patterns in volatility of futures prices in more detail. Specifically, we estimate a functional relation between the futures price volatility and the one-day lagged slope of the forward curve. Following the definition of conditional sample moments, the time series of the slope of the forward curve is constructed as a logarithm of the ratio of the futures price of the third shortest maturity in months available on any day $t, P(t, 3)$, to the future price of the shortest maturity, $P(t, 1)$, available on the same day. We start by using lagged slope as the only explanatory variable for realized volatility

$$
|R(t, T)|=\alpha_{T}+\beta_{T} \ln \left[\frac{P(t-1,3)}{P(t-1,1)}\right]+\varepsilon_{T}(t) .
$$

Note that the relation (1) can potentially yield different information than that contained in Figures 1-3, which were obtained by simply splitting the sample based on the slope of the forward curve, since we are now estimating a different functional form. The term structure of $\beta_{T}$ as well as the corresponding $t$-statistics are shown for different commodities in Figures 4 through 6 . We also report these results in Tables 1 through 3 for $T$ equal to 1,5 , and 10 months. The negative sign of $\beta_{T}$ for all times to maturity (most of them in the case of the HU contract) is a common feature across contracts. This result seems to be at odds with the relations shown in Figures 1 through 3, where volatility conditional on backwardation is mostly higher than the unconditional volatility. The apparent inconsistency becomes less puzzling in light of the intuition of the model we present below. In particular, our theoretical results motivate one to look for a non-monotone relationship between the volatility of future prices and the slope of their term structure. For that we decompose the lagged slope into positive and negative parts and use them as separate explanatory variables (i.e., use a piecewise linear regression on the slope of the term structure),

$$
|R(t, T)|=\alpha_{T}+\beta_{1, T}\left(\ln \left[\frac{P(t-1,3)}{P(t-1,1)}\right]\right)^{+}+\beta_{2, T}\left(\ln \left[\frac{P(t-1,3)}{P(t-1,1)}\right]\right)^{-}+\varepsilon_{T}(t),
$$

where $(X)^{ \pm}$denotes the positive (negative) part of $X$ respectively. Figures 4 through 6 
as well as Tables 1 through 3 illustrate our results. Both $\beta_{1, T}$ and $\beta_{2, T}$ are statistically and economically significant for most maturities and across contracts. More importantly, $\beta_{1, T}$ and $\beta_{2, T}$ differ in sign: $\beta_{1, T}$ are positive and $\beta_{2, T}$ are negative. Therefore, the relation between the volatility of futures prices and the slope of the term structure of prices is nonmonotone and has a "V-shape": conditional volatility declines as a function of the slope when the latter is negative, and increases when the latter is positive.

We perform several robustness checks. Using the square of daily price changes instead of the absolute value, i.e., estimating conditional variance instead of conditional volatility, leads to very similar conclusions. In most cases, both $\beta_{1, T}$ and $\beta_{2, T}$ remain statistically significant for CL and HO contracts, but only marginally significant for HU contract.

As a final robustness check we split our sample into pre- and post-Gulf war sub-samples. We perform the same analysis as in the case of the full sample on the post-Gulf war subsample. We find the same "V-shape" in the relationship between the volatility of futures prices and the slope of the term structure of prices for CL and HO contracts. The "V-shape" disappears in the post-Gulf war sub-sample in the case of HU contracts.

\section{The Model}

In this section we present our model for spot prices and derive futures prices.

\subsection{Setup}

We consider a continuous-time infinite-horizon economy. We focus on a competitive industry populated by firms using an identical production technology. Production in the economy is done by means of a production function that exhibits constant returns to scale

$$
Q_{t}=X K_{t}
$$


where $K_{t}$ is capital and $X$ is the productivity of capital which is assumed to be constant. For the sake of simplicity we will assume below that $X=1$. Our results can be easily adjusted to accommodate the case when $X$ is a stochastic process. Firms can adjust their capital stock according to

$$
d K_{t}=\left(i_{t}-\delta\right) K_{t} d t
$$

where $i_{t}$ is the investment rate and $\delta$ is the capital depreciation assumed to be a nonnegative constant. We assume that investment is irreversible, i.e., $i_{t} \geq 0$, and the rate of investment is bounded, i.e., $i_{t} \in[0, \bar{i}]$. These investment frictions give rise to the dynamic properties of futures prices investigated below.

Firms sell their output in the spot market at price $S_{t}$. We assume that financial markets are complete and the firms' objective is to maximize their market value, which in turn is given

$$
V_{0}=\mathrm{E}_{0}\left[\int_{0}^{\infty} e^{-r t} S_{t} Q_{t} d t\right]
$$

We assume that the expected value is computed under the risk-neutral measure and the risk-free rate $r$ is constant.

The consumers in the economy are represented by the demand curve

$$
Q_{t}=Y_{t}^{-1} S_{t}^{-\frac{1}{\gamma}}, \quad Q_{t} \in(0, \infty)
$$

where unexpected changes in $Y_{t}$ represent demand shocks. We assume that $Y_{t}$ follows a geometric Brownian motion process

$$
\frac{d Y_{t}}{Y_{t}}=\mu_{Y} d t+\sigma_{Y} d W_{t}
$$

We also assume that $\gamma>1$. Results for the case of $\gamma \leq 1$ are analogous and are available from the authors.

Since our model is formulated under the risk-neutral probability measure, while the empirical observations are made under the "physical" probability measure, one has to make 
an explicit assumption about the relation between these two measures, i.e., about the risk premium associated with the shock process $d W_{t}$. To keep our specification as simple as possible, we assume that the risk premium is constant, i.e., the drift of the demand shock $Y_{t}$ under the "physical" probability measure is equal to $\mu_{Y}+\lambda$, where $\lambda$ is an additional parameter of the model.

\subsection{Equilibrium Investment and Prices}

Following Lucas and Prescott (1971), we characterize the equilibrium investment policy as a solution of the social planner's problem. Specifically, we define the surplus function ${ }^{3}$

$$
U\left(Y_{t}, Q_{t}\right)=\int_{1}^{Q_{t}} S_{t}(q) d q=Y_{t}^{-\gamma} \int_{1}^{Q_{t}} Q^{-\gamma} d Q=Y_{t}^{-\gamma} \frac{Q_{t}^{1-\gamma}-1}{1-\gamma}
$$

The social planner maximizes total surplus net of investment costs

$$
\max _{i_{t} \in[0, \bar{i}]} \mathrm{E}_{0}\left[\int_{0}^{\infty} e^{-r t}\left(Y_{t}^{-\gamma} \frac{K_{t}^{1-\gamma}}{1-\gamma}-i_{t} K_{t}\right) d t\right]
$$

subject to the dynamics of the demand curve and the capital accumulation rule

$$
\begin{gathered}
d K_{t}=\left(i_{t}-\delta\right) K_{t} d t, \\
\frac{d Y_{t}}{Y_{t}}=\mu_{Y} d t+\sigma_{Y} d W_{t}, \\
K_{t} \geq 0, \quad \forall t \geq 0 .
\end{gathered}
$$

The details of the solution are provided in the Appendix. The equilibrium investment policy satisfies

$$
i_{t}^{*}=\left\{\begin{array}{l}
\bar{i}, \omega_{t} \leq \omega^{*} \\
0, \omega_{t}>\omega^{*}
\end{array}\right.
$$

where $\omega_{t}=\ln \left(K_{t} Y_{t}\right)$ and the no-investment boundary $\omega^{*}$ is given in the Appendix. To make sure that $\omega^{*}$ is finite, we impose a restriction on model parameters, (A.14).

\footnotetext{
${ }^{3}$ Starting the integration at 1 is inconsequential for our analysis. Recall that $Q$ is restricted to be strictly positive guaranteeing a well defined objective function in equation (9). Starting the integration at any positive point below one would only add a constant to the objective function in equation (9) not affecting the first order conditions.
} 
A formal proof that the proposed investment policy arises in competitive equilibrium is technically demanding (see the Appendix) and we need to impose an additional non-trivial restriction on parameter values:

$$
\frac{\sigma_{Y}^{2} \gamma^{2}}{2}+\gamma \mu^{+}-(r+\delta)<0
$$

where we define $\mu^{-}=\delta-\mu_{Y}+\frac{1}{2} \sigma_{Y}^{2}$ and $\mu^{+}=\bar{i}-\mu^{-}$. The above restriction on parameters plays an important role in calibration and is easy to overlook without a rigorous verification of optimality.

The evolution of the state variable $\omega_{t}$ under the risk-neutral measure is governed by

$$
d \omega_{t}=\left[i_{t}^{*}-\mu^{-}\right] d t+\sigma_{Y} d W_{t}
$$

As long as $0<\mu^{-}<\bar{i}, \omega_{t}$ has a stationary long-run distribution

$$
p(\omega)=\frac{2 \mu^{-}\left(\bar{i}-\mu^{-}\right)}{\bar{i} \sigma_{Y}^{2}} \exp \left(\frac{2}{\sigma_{Y}^{2}}\left(\omega-\omega^{*}\right)\left[i_{t}^{*}-\mu^{-}\right]\right)
$$

The details of the derivation are provided in the Appendix. The spot price process of firms' output is related to the equilibrium capital stock by the inverse demand curve

$$
S_{t}=\left[K_{t} Y_{t}\right]^{-\gamma}=e^{-\gamma \omega_{t}}
$$

It is worth mapping our general investment constraint model to the oil industry. Oil $(Q)$ is the output produced using physical capital $K$ (e.g., refineries, pipes). Implicitly we are assuming there is an infinite supply of underground oil, and production is constrained by the existing capital stock $K$. This supply of capital and consequently of oil-output leads to price fluctuations in response to demand shocks. Futures prices (volatility) depend on anticipated future production which depends on the degree to which investment is constrained.

\subsection{Futures Prices}

The futures contract is a claim on the good which is sold on the spot market at prevailing spot price $S_{t}$. The futures price is computed as the conditional expectation of the spot price 
under the risk-neutral measure:

$$
P(t, T)=\mathrm{E}_{t}\left[S_{t+T}\right]=\mathrm{E}_{t}\left[e^{-\gamma \omega_{t+T}}\right], \quad \forall T \geq 0 .
$$

where $P(t, T)$ denotes the price of a futures contract at time $t$ with maturity date $\tau=t+T$. According to the Feynman-Kac Theorem (e.g., Karatzas and Shreve, 1991, Thm. 4.4.2), the futures prices of a contract maturing at time $\tau$ can be equivalently characterized as a solution of a partial differential equation

$$
\frac{\partial f}{\partial t}+\left(i^{*}(\omega)-\mu^{-}\right) \frac{\partial f}{\partial \omega}+\frac{1}{2} \sigma_{Y}^{2} \frac{\partial^{2} f}{\partial \omega^{2}}=0
$$

with a terminal condition

$$
f\left(\tau, \omega_{\tau}\right)=e^{-\gamma \omega_{\tau}} .
$$

Then, $P(t, T)=f\left(t, \omega_{t}\right)$. Since no analytical solution exists for the above equation, we solve it numerically using a finite-difference scheme.

\section{Estimation and Numerical Simulation}

In this section we study how well our model can replicate quantitatively the key features of the behavior of futures prices reported in Section 1. We first estimate the model's parameters using simulated method of moments. Our procedure is similar in spirit, but somewhat different technically, from those proposed in Lee and Ingram (1991) and Duffie and Singleton (1993). We then discuss additional implications of the model.

\subsection{Simulated Moments Parameter Estimation Estimation Procedure}

Our goal is to estimate a vector of structural parameters, $\theta \equiv\left\{\gamma, \mu_{Y}, \sigma_{Y}, \bar{i}, r, \delta, \lambda\right\}$. We do this using a classical minimum distance (CMD) method, which requires matching a set of functions of structural parameters with the corresponding set of empirical estimates. 
Our procedure can be equivalently viewed as a generalized method of moments (GMM) estimation, since all the functions we consider can be expressed as sample moments. Let $x_{t}$ be the vector-valued process of historical futures prices and output and consider a function of the observed sample $F_{T}(x)$, where $T$ is the sample length. The statistic $F_{T}(x)$ could represent a collection of sample moments or even a more complicated estimator, such as the slope coefficients in a regression of volatility on the term structure as in (1). Assume that as the sample size $T$ increases, $F_{T}(x)$ converges in probability to a limit $M(\theta)$, which is a function of structural parameters. Since many of the useful population moments cannot be computed analytically, we estimate them using Monte Carlo simulation. In particular, let $m_{S}(\theta)=\frac{1}{S} \sum_{s=1}^{S} F_{T}\left(x^{s} ; \theta\right)$ represent the estimate of $M(\theta)$ based on $S$ independent model based statistics, where $x^{s}$ represents a vector valued process of simulated futures prices and output of length $T$ based on simulating the model at parameter values, $\theta .{ }^{4}$ Let $G_{S}(x, \theta)=m_{S}(\theta)-F_{T}(x)$, denote the difference between the estimated theoretical mean of the statistic $F$ and it's observed (empirical) value. Under appropriate regularity conditions, it can be shown that as the size of the sample, $T$, and the number of simulations $S$ increase to infinity, the CMD (GMM) estimate of $\theta$,

$$
\theta_{S}=\arg \min _{\theta} J_{T}=\arg \min _{\theta} G_{S}(x, \theta)^{\prime} W_{T} G_{S}(x, \theta)
$$

will be a consistent estimator of $\theta$. The matrix $W_{T}$ in the above expression is positive definite and assumed to converge in probability to a deterministic positive definite matrix $W$.

Assume that $V$ is the asymptotic variance-covariance matrix of $F_{T}(x ; \theta)$. Then, if we use the efficient choice of the weighting matrix, $W=V^{-1}$, the estimator $\theta_{S}$ is asymptotically normal, with mean $\theta$ and covariance matrix $\left(D^{\prime} V^{-1} D\right)^{-1}$, where $D=\nabla_{\theta} M(\theta)$.

\footnotetext{
${ }^{4}$ Specifically, for any given value of $\theta$, we draw $S$ realizations of the state variable $\omega_{t}$ from its long-run steady-state distribution (which itself depends on model parameters and is given by (13)). Then, for each set of initial conditions, we simulate a path of the state variable of the same length as the historical sample and evaluate the function $F(x, \theta)$ for each simulated path of the economy.
} 
We perform estimation in two stages. During the first stage, we use an identity matrix for the weighting matrix $W$. During the second stage, the weighting matrix is set equal to the inverse of the estimated covariance matrix: $W=V_{S}^{-1}$, where $V_{S}$ is the sample based covariance matrix of $F_{T}\left(x^{s} ; \theta\right)$. To compute standard errors, we use as an estimate for $D$, $D_{S}=\nabla_{\theta} m_{S}(\theta)$

We estimate the vector of seven model parameters, $\theta$, by matching the unconditional properties of futures prices, specifically, the unconditional mean and volatility of daily percent price changes for futures of various maturities as well as the mean, volatility, and the 30-day autoregressive coefficient of the slope of the forward curve. We use crude oil futures prices as a benchmark. Reproducing unconditional properties of futures prices with a simple singlefactor model is a nontrivial task, as we discuss below. However, the most novel implications of our model have to do with the conditional nature of the relation between the volatility of futures prices and the slope of the term structure. With this in mind, we choose model parameters to match the unconditional level of futures price volatility and then evaluate the quality of model predictions based on the conditional moments, which were not used in estimation.

\section{Identification}

Not all of the model parameters can be independently identified from the data we are considering. In this subsection we discuss the relations between structural parameters and observable properties of our model economy, which suggest which of the structural parameters can be identified and what dimensions of empirical data are likely to be most useful for estimation.

First, we calibrate the risk free rate. The risk free rate is determined by many factors outside of the oil industry and consequently it would not be prudent to estimate it solely based on oil-price data. Also, it is clear by inspection that the risk-free rate is not identified 
by our model. It does not affect any of the moments we consider in our estimation and only appears in the constraint on model parameters in equation (A.7). Therefore, at best, futures price data can only impose a lower bound on the level of the risk-free rate, as implied by (A.7). Given all of the above considerations, we set the risk free rate at $2 \%{ }^{5}$

Next, consider a simple re-normalization of the structural parameters. Since futures prices in our model depend solely on the risk-neutral dynamics of the spot price, which in turn depends only on $\gamma \omega_{t}$, futures prices are determined by $\tilde{\omega}_{t}=\gamma \omega_{t}$, which evolves according to

$$
d \tilde{\omega}_{t}=\left[\gamma \bar{i} 1_{\left[\omega \leq \omega^{*}\right]}-\gamma \mu^{-}\right] d t+\gamma \sigma_{Y} d W_{t}
$$

where $1_{[\cdot]}$ is an indicator function. Since we normalize the productivity parameter in (3) to one, only relative prices are informative, and therefore we can ignore the dependence of $\omega^{*}$ on structural parameters. Thus, the risk-neutral dynamics of futures prices is determined by only three combinations of five structural parameters: $\gamma \mu^{-}, \gamma \bar{i}$, and $\gamma \sigma_{Y}$. Therefore, we cannot identify all the model parameters separately from the futures data alone.

We obtain an additional identifying condition from the oil consumption data. As documented in Cooper (2003), world crude oil consumption increased by 46 per cent per capita from 1971 to 2000, implying an average growth rate of approximately $1.25 \%$. Individual growth rates vary for the 23 three countries considered by Cooper, typically falling between -3 to $3 \%$. For the US, the reported growth rate averaged $-0.7 \%$ which we attempt to equate with the expected growth rate of oil consumption, $g_{C}$, implied by the model

$$
g_{C}=\bar{i} \operatorname{Pr}\left(\omega \leq \omega^{*}\right)-\delta=\frac{1}{2} \sigma_{Y}^{2}-\left(\lambda+\mu_{Y}\right),
$$

where $\operatorname{Pr}\left(\omega \leq \omega^{*}\right)=\int_{-\infty}^{\omega^{*}} p^{+}(\omega) d \omega=(\bar{i})^{-1} \mu^{-}$is the unconditional probability that $\omega$ is below the investment trigger.

\footnotetext{
${ }^{5}$ Our results regarding the ' $\mathrm{V}$ ' shape response in prices are not affected by this choice.
} 
Finally, to estimate the risk premium $\lambda$, we use average historical daily returns on fully collateralized futures positions (we use three-month contracts). We are thus left with five independent identifying restrictions on six structural parameters. Following Gomes (2001), we fix the depreciation rate of capital at $\delta=0.12$ per year and do not infer it from futures prices. We estimate the remaining five parameters.

\section{Parameter Estimates}

Our estimated parameter values and the corresponding standard errors are summarized in Table 4. The first parameter value in the table, $\gamma=3.42$, implies that the price elasticity of demand in our model is -0.29 . Cooper (2003) reports estimates of short-run and long-run demand elasticity for a partial adjustment demand equation based on US data of -0.06 and -0.45 respectively. In our model, there is no distinction between short-run and longrun demand, as demand adjustments are assumed to be instantaneous. Our estimate falls half-way between the two numbers reported in Cooper (2003) for the US and is close to the average of the long-run elasticity estimates reported for all 23 countries considered in that study, which is -0.2 .

Our second parameter is $\bar{i}$, the maximum investment rate in the model. This variable parameterizes the investment technology used by the firms. While it is difficult to make direct empirical comparisons, the upper bound of $\bar{i}=0.14$ would allow for a plausible range of realized annual investment rates.

The average growth rate of demand is close to zero, as is the market price of risk. For comparison, the average annualized change in futures prices is approximately $2.8 \%$ in the data, which falls within the $95 \%$ confidence interval of the model's prediction. The volatility of demand shocks is not directly observable. The estimated value of $\sigma_{Y}$, together

with the demand elasticity parameter $\gamma^{-1}$ imply annualized volatility of the spot price of approximately $40 \%$, which is closed to the observed price volatility of short-maturity futures 
contracts.

\subsection{Results and Discussion}

We first illustrate the fit of the model by plotting the term structure of unconditional futures price volatility (to facilitate comparison with empirical data, we express our results as daily values, defined as annual values scaled down by $\sqrt{252}$ ). We chose model parameters, as summarized in Table 4, to match the behavior of crude oil futures. Figure 7 compares the volatility of prices implied by our choice of parameters to the empirical estimates. Our model seems capable of reproducing the slow-decaying pattern of futures price volatility. This feature of the data presents a challenge to simple storage models, as discussed in Routledge et. al. (2000). To see why it may not be easy to reproduce the slow-decaying pattern of unconditional volatilities in a simple single-factor model, consider a reduced-form model in which the logarithm of the spot price process follows a continuous-time $\operatorname{AR}(1)$ process (Ornstein-Uhlenbeck process). Specifically, assume that the spot price is given by

$$
S_{t}=e^{y_{t}}
$$

and under the risk-neutral probability measure $y_{t}$ follows

$$
d y_{t}=\theta_{y}\left(\bar{y}-y_{t}\right) d t+\sigma_{y} d W_{t}
$$

where $\theta_{y}$ is the mean-reversion coefficient and $\bar{y}$ is the long-run mean of the state variable. According to this simple model, the unconditional volatility of futures price changes is an exponential function of maturity $\tau$ :

$$
\sigma^{2}(\tau)=\sigma_{y}^{2} e^{-2 \theta \tau}
$$

To compare the term structure of unconditional volatility implied by this model to the one generate by our model, we calibrate parameters $\theta_{y}$ and $\sigma_{y}$ so that the simple model exhibits 
the same volatility of the spot price and the same 30-day autocorrelation of the basis as our model. Figure 7 shows that, as expected, unconditional volatility implied by the simple model above decays too fast relative to our model and data.

The main qualitative distinction between the properties of our model and those of basic storage models is in the conditional behavior of futures volatility. As we demonstrate in Section 2, the empirical relation between the volatility of futures prices and the slope of the term structure of prices is non-monotone and has a pronounced "V-shape". Intuitively, we would expect our model to exhibit this pattern. When the state variable $\omega_{t}$ is far away from the investment trigger $\omega^{*}$, one of the investment constraints is binding and can be expected to remain binding for some time. If the capital stock $K_{t}$ is much higher than its optimal level, given the current level of demand, firms find it optimal to postpone investment and the irreversibility constraint binds. On the other hand, when $K_{t}$ is much lower than the optimal level, firms invest at the maximum possible rate and the investment rate constraint binds. In either case, the supply of the commodity is relatively inelastic and futures prices are relatively volatile. The further $\omega_{t}$ travels away from the investment trigger, the larger the effect on volatility of long-maturity futures. At the same time, it is precisely when $\omega_{t}$ is relatively far away from the investment trigger $\omega^{*}$, when the absolute value of the slope of the term structure of futures prices is large. This is to be expected. All prices in our model are driven by a single mean-reverting stationary state variable, and since futures prices of longer-maturity contracts are less sensitive to the current value of the state variable than the spot price, the slope of the forward curve tends to be large when the state variable is far away from its long-run average value. The latter, in turn, is not far from $\omega^{*}$, given that $\omega_{t}$ reverts to $\omega^{*}$. Thus, our model predicts that the volatility of futures prices should exhibit a "V-shape" as a function of the slope of the term structure of futures prices.

It should be clear from the above discussion that the critical feature of the model is not 
the precise definition of the production function, but rather the variable-elasticity property of the supply side of the economy. The "V-shape" pattern in volatilities is due to the fact that supply can adjust relatively easily in response to demand shocks when the output is close to the optimal level, but supply is relatively inelastic when the output level is far from the optimum.

We now report the quantitative properties of the model. The long-run average of the slope of the forward curve, $\ln \left[\frac{P(t-1,3)}{P(t-1,1)}\right]$, is 0.008 in the model, compared to the empirical value of -0.0151 . Both values are statistically indistinguishable from zero. The long-run standard deviation of the slope in the model, which equals 0.017 , is half the empirical value of 0.0326 . The 30-day autocorrelation coefficient of the slope implied by the model is equal to 0.72 , as compared to the value of 0.72 in the data. Overall, our model fits the basic behavior of the slope of the forward curve quite well. Table 5 shows the estimates of linear and piece-wise linear specifications of conditional variance of futures price changes (2) implied by the model for one-, five-, and ten-month futures. The coefficients of the linear regressions are negative and close in magnitude to their empirical counterparts. Such a negative relation between conditional volatility of futures prices and the basis would typically be interpreted as supportive of simple storage models. Note, however, that our model without storage can reproduce the same kind of relation. Our model, however, has a further important implication: the linear model is badly misspecified, since the theoretically predicted relation is non-monotone. Our piece-wise linear specification produces coefficients $\beta_{1, T}$ and $\beta_{2, T}$ that agree with their empirical counterparts in sign but differ in magnitude. Given the extremely streamlined nature of our model (e.g., the basis is a sufficient statistic for conditional volatility), this should not be surprising. Also, it is important to keep in mind that we did not target the volatility-basis relation in our estimation of structural parameters. The entire distribution of regression coefficients across maturities of the futures contracts is 
shown in Figure 8. Finally, Figure 9 helps visualize the "V-shape" pattern.

In order to understand the sensitivity of our results to the baseline parameters summarized in Table 4, we compute elasticities of basic statistics of the model output with respect to these parameters. Each elasticity is calculated by simulating the model twice: with a value of the parameter of interest ten percent of one standard deviation below (above) its baseline value. Next, the change in the moment is calculated as the difference between the results from the two simulations. This difference is then divided by the change in the underlying structural parameter between the two simulations. Finally, the result is then multiplied by the ratio of the baseline structural parameter to the baseline moment. The elasticities are reported in Table 6 .

An increase in the demand volatility, $\sigma_{Y}$, or in the elasticity of the inverse demand curve, $\gamma$, leads to an increase in the volatility of the spot price, which equals $\gamma^{2} \sigma_{Y}^{2}$. As one would expect, volatility of futures prices of various maturities increases as well. Qualitatively, both of the parameters $\sigma_{Y}$ and $\gamma$ affect the level of the unconditional volatility curve plotted in Fig. 7. However, the demand volatility has strong positive effect on the expected growth rate of oil consumption since it increases the long-run growth rate of the level of the demand curve, $Y_{t}^{-1} \cdot \gamma$ has no such effect.

The constraint on the investment rate $\bar{i}$ has no effect on the volatility of the spot price. However, it affects volatility of futures prices. A higher value of $\bar{i}$ allows capital stock to adjust more rapidly in response to positive demand shocks, thus reducing the impact of demand shocks on the future value of the spot price and therefore lowering the volatility of futures prices. We thus see that $\bar{i}$ effectively controls the slope of the term structure of volatility, higher values of $\bar{i}$ imply a steeper term structure. $\bar{i}$ has no effect on the expected growth rate of oil consumption, in agreement with Eq. (17).

An increase in the unconditional mean of the demand shock, $\mu_{Y}$, has little affect on the 
level of futures price volatility. This is not surprising given the role $\mu_{Y}$ plays in the evolution of the state variable $\omega_{t}$. An increase in $\mu_{Y}$ raises the drift of $\omega_{t}$ uniformly. The impact of this on the volatility of futures price is ambiguous and depends on the relative magnitude of the drift of $\omega_{t}$ above, $\mu^{-}$, and below, $\mu^{+} \equiv \bar{i}-\mu^{-}$, the investment threshold $\omega^{*}$. By symmetry considerations, if $\mu^{+}=\mu^{-}$, an infinitesimal change in $\mu_{Y}$ has no impact on the volatility of futures prices. Under the calibrated parameter values, $\mu^{-}=0.11$ and $\mu^{+}=0.025$ and futures volatility is not very sensitive to $\mu_{Y}$. The same is true for the risk premium, $\lambda$. Both $\mu_{Y}$ and $\lambda$ have strong negative effect on $g_{C}$ in agreement with Eq. (17).

In general, the affect of model parameters on the slope of the forward curve is difficult to interpret intuitively and depends on the chosen parameter values. However, the fact that the moments of the slope have different sensitivities to various model parameters makes them useful in estimating these parameters.

\section{Conclusions}

This paper contributes along two dimensions. First, we show that volatility of future prices has a "V-shape" relationship with respect to the slope of the term structure of futures prices. Second, we show that such volatility patterns arise naturally in models that emphasize investment constraints and, consequently, time-varying supply-elasticity as a key mechanism for price dynamics. Our empirical findings seem beyond the scope of simple storage models, which are currently the main focus of the literature, and point towards investigating alternative economic mechanisms, such as the one analyzed in this paper. Future work will entail a model that nests both storage and investment in an attempt to isolate their quantitative effects. 


\section{References}

Brennan, M., 1991, "The Price of Convenience and the Valuation of Commodity Contingent Claims" in D. Lund and B. Oksendal (eds.), Stochastic Models and Option Models, Elsevier Science Publishers, 1991.

Brennan, M. and E. Schwartz, 1985, "Evaluating Natural Resource Investments," Journal of Business 58, 135-157.

Casassus, J., P. Collin-Dufresne and B. Routledge, 2004, "Equilibrium Commodity Prices with Irreversible Investment and Non-Linear Technologies", CMU working paper.

Cooper, John C. B., 2003, "Price Elasticity of Demand for Crude Oil: Estimates for 23 Countries," OPEC Review 27, 1-8.

Deaton, A. and G. Laroque, 1992, "On the Behavior of Commodity Prices," The Review of Financial Studies 59, 1-23.

Deaton, A. and G. Laroque, 1996, "Competitive Storage and Commodity Price Dynamics," Journal of Political Economy 104, 896-923.

Duffie, Darrell and Ken Singleton, 1993, "Simulated moments estimation of Markov models of asset prices," Econometrica 61, 929-952.

Gomes, Joao F., 2001, "Financing investment," American Economic Review 91, 1263-1285.

Kaldor, N., 1939, "Speculation and Economic Stability," The Review of Economic Studies $7,1-27$.

Karatzas, I. and S.E. Shreve, 1991, Brownian Motions and Stochastic Calculus, SpringerVerlag. 
Kogan, L., 1999, Doctoral Dissertation, Sloan School of Management, MIT, Boston, MA.

Lee, B. and B. Ingram, 1991, "Simulation estimation of time series models", Journal of Econometrics 47, 197-205.

Lucas, R., and E. Prescott, 1971, "Investment under Uncertainty," Econometrica 39, 659681.

Routledge, B., D. Seppi and C. Spatt, 2000, "Equilibrium Forward Curves for Commodities," Journal of Finance 55, 1297-1338.

Schwartz, E., 1997, "The Stochastic Behavior of Commodity Prices: Implications for Valuation and Hedging," Journal of Finance 52, 923-973.

Telser, L.G., 1958, "Futures Trading and the Storage of Cotton and Wheat," Journal of Political Economy 66, 133-144.

Williams, J. and B. Wright, 1991, Storage and Commodity Markets. Cambridge, England: Cambridge University Press.

Working, H., 1948, "Theory of the Inverse Carrying Charge in Futures Markets," Journal of Farm Economics 30, 1-28.

Working, H., 1949, "The Theory of the Price of Storage," American Economic Review 39, $1254-1262$. 


\section{Appendix}

\section{A. Central Planner's Problem}

To characterize the solution of the social planner's problem, we define the value function $J\left(K_{t}, Z_{t}\right)$ as

$$
J\left(K_{t}, Y_{t}\right)=\max _{i_{s} \in[0, \bar{i}]} \mathrm{E}_{t}\left[\int_{t}^{\infty} e^{-r(s-t)}\left(Y_{s}^{-\gamma} \frac{K_{s}^{1-\gamma}}{1-\gamma}-i_{s} K_{s}\right) d s\right]
$$

The Hamilton-Jacobi-Bellman (HJB) equation for $J\left(K_{t}, Z_{t}\right)$ takes the form

$$
\max _{i \in[0, \bar{i}]}\left[i K J_{K}-i K\right]+\frac{\sigma_{Y}^{2}}{2} Y^{2} J_{Y Y}+\mu_{Y} Y J_{Y}-\delta K J_{K}+Y^{-\gamma} \frac{K^{1-\gamma}}{1-\gamma}-r J=0 .
$$

The solution to the above equation consists of the value function and endogenously determined no-investment boundary determined by $J_{K}=1$. When $J_{K} \geq 1$ the investment is made at a maximum rate $i=\bar{i}$, and no investment is made when $J_{K}<1$.

We make the following change of variables in HJB equation

$$
k=\ln K, y=\ln Y,
$$

to obtain

$$
\max _{i_{t} \in[0, \bar{i}]}\left[i J_{k}-i e^{-y} e^{k+y}\right]+\frac{\sigma_{Y}^{2}}{2} J_{y y}+\left(\mu_{Y}-\frac{\sigma_{Y}^{2}}{2}\right) J_{y}-\delta J_{k}+\frac{e^{-y} e^{(1-\gamma)(k+y)}}{1-\gamma}-r J=0 .
$$

We will look for the solution in the form

$$
J(k, y)=\frac{e^{-y} f(\omega)}{1-\gamma},
$$

where $\omega=k+y$. We have

$$
\begin{aligned}
J_{k} & =\frac{e^{-y} f^{\prime}}{1-\gamma}, \\
J_{y} & =\frac{e^{-y}}{1-\gamma}\left(f^{\prime}-f\right), \\
J_{y y} & =\frac{e^{-y}}{1-\gamma}\left(f^{\prime \prime}-2 f^{\prime}+f\right),
\end{aligned}
$$


and the ODE for $f$ takes the form

$$
\max _{i \in[0, \bar{i}]}\left[i f^{\prime}-i(1-\gamma) e^{\omega}\right]+\frac{\sigma_{Y}^{2}}{2} f^{\prime \prime}+\left(\mu_{Y}-\delta-\frac{3 \sigma_{Y}^{2}}{2}\right) f^{\prime}+\left(\sigma_{Y}^{2}-\mu_{Y}-r\right) f+e^{(1-\gamma) \omega}=0 .
$$

We will look for a solution of (A.1) of the form

$$
f(\omega)=\left\{\begin{array}{c}
A \exp \left(\varkappa_{-}\left(\omega-\omega^{*}\right)\right)+B \exp ((1-\gamma) \omega), \omega \geq \omega^{*}, \\
\bar{A} \exp \left(\bar{\varkappa}_{+}\left(\omega-\omega^{*}\right)\right)+\bar{B} \exp ((1-\gamma) \omega)+C \exp (\omega), \omega<\omega^{*},
\end{array},\right.
$$

for all $\gamma^{\prime}$ s, where $\omega^{*}$ is the no-investment boundary in terms of the new state variable $\omega$. Substituting these solutions into ODE (A.3) yields quadratic equations on $\varkappa_{ \pm}$and $\bar{\varkappa}_{ \pm}$

$$
\begin{array}{r}
\frac{\sigma_{Y}^{2}}{2} \varkappa_{ \pm}^{2}-\left(\delta+\frac{3 \sigma_{Y}^{2}}{2}-\mu_{Y}\right) \varkappa_{ \pm}+\left(\sigma_{Y}^{2}-\mu_{Y}-r\right)=0, \\
\frac{\sigma_{Y}^{2}}{2} \bar{\varkappa}_{ \pm}^{2}-\left(\delta-\bar{i}+\frac{3 \sigma_{Y}^{2}}{2}-\mu_{Y}\right) \bar{\varkappa}_{ \pm}+\left(\sigma_{Y}^{2}-\mu_{Y}-r\right)=0,
\end{array}
$$

and constants $B, \bar{B}$, and $C$

$$
\begin{aligned}
B & =\frac{1}{r+\gamma \mu_{Y}+(1-\gamma) \delta-\frac{\gamma(1+\gamma)}{2} \sigma_{Y}^{2}}, \\
\bar{B} & =\frac{1}{r+\gamma \mu_{Y}-(1-\gamma)(\bar{i}-\delta)-\frac{\gamma(1+\gamma)}{2} \sigma_{Y}^{2}}, \\
C & =\frac{(1-\gamma) \bar{i}}{\bar{i}-\delta-r} .
\end{aligned}
$$

For the quadratic equation on $\varkappa$ to have one negative root, we impose that

$$
r+\mu_{Y}-\sigma_{Y}^{2}>0
$$

As of now we have determined constants $B, \bar{B}, C, \varkappa_{-}$and $\bar{\varkappa}_{+}$, and we are left to find four more parameters, constants $A, \bar{A}$, and, finally, the no-investment boundary $\omega^{*}$ in both cases. We have three boundary conditions to determine these constants. The first two boundary conditions ensure continuity of $f(\omega)$ and its first derivative across the noinvestment boundary

$$
\begin{gathered}
f\left(\omega^{*}-0\right)=f\left(\omega^{*}+0\right), \\
f^{\prime}\left(\omega^{*}-0\right)=f^{\prime}\left(\omega^{*}+0\right) .
\end{gathered}
$$


The investment optimality condition, $J_{K}=1$, which takes the following form in terms $\omega$

$$
f^{\prime}\left(\omega^{*}\right)=(1-\gamma) e^{\omega^{*}}
$$

is the third and the final one. Substituting equation (A.4) into the boundary conditions we obtain the following system of equations

$$
\left\{\begin{array}{l}
\bar{A}-A=(B-\bar{B}) e^{(1-\gamma) \omega^{*}}-C e^{\omega^{*}} \\
\bar{\varkappa}_{+} \bar{A}-\varkappa_{-} A=(1-\gamma)(B-\bar{B}) e^{(1-\gamma) \omega^{*}}-C e^{\omega^{*}} \\
\varkappa_{-} A+(1-\gamma) B e^{(1-\gamma) \omega^{*}}=(1-\gamma) e^{\omega^{*}}
\end{array}\right.
$$

Solving last two equations in both systems we find the unique solution

$$
\begin{aligned}
A & =\frac{\bar{\varkappa}_{+}+\gamma-1}{\bar{\varkappa}_{+}+\left|\varkappa_{-}\right|}(\bar{B}-B) e^{(1-\gamma) \omega^{*}}+\frac{\bar{\varkappa}_{+}-1}{\bar{\varkappa}_{+}+\left|\varkappa_{-}\right|} C e^{\omega^{*}}, \\
\bar{A} & =\frac{\gamma-\left|\varkappa_{-}\right|-1}{\bar{\varkappa}_{+}+\left|\varkappa_{-}\right|}(\bar{B}-B) e^{(1-\gamma) \omega^{*}}-\frac{\left|\varkappa_{-}\right|+1}{\bar{\varkappa}_{+}+\left|\varkappa_{-}\right|} C e^{\omega^{*}}, \\
e^{\gamma \omega^{*}} & =\frac{\left(1-\gamma+\left|\varkappa_{-}\right|\right) \bar{\varkappa}_{+} B+\left(1-\gamma-\bar{\varkappa}_{+}\right)\left|\varkappa_{-}\right| \bar{B}}{(1-\gamma)\left(\bar{\varkappa}_{+}+\left|\varkappa_{-}\right|\right)+\left|\varkappa_{-}\right|\left(\bar{\varkappa}_{+}+\gamma-1\right) C} .
\end{aligned}
$$

So far we assumed that the optimal policy is characterized by a finite value of $\omega^{*}$ : it is optimal to invest when $\omega$ is sufficiently low, and it is optimal not to invest when $\omega$ is sufficiently high. To make sure that our assumption is correct, we need to impose a restriction on model parameters: we assume that

$$
\frac{\left(1-\gamma+\left|\varkappa_{-}\right|\right) \bar{\varkappa}_{+} B+\left(1-\gamma-\bar{\varkappa}_{+}\right)\left|\varkappa_{-}\right| \bar{B}}{(1-\gamma)\left(\bar{\varkappa}_{+}+\left|\varkappa_{-}\right|\right)+\left|\varkappa_{-}\right|\left(\bar{\varkappa}_{+}+\gamma-1\right) C}>0 .
$$

\section{B. Stationary long-run distribution of $\omega_{t}$}

In this appendix we calculate the stationary long-run distribution of the state variable $\omega$, dynamics of which can be written as

$$
d \omega_{t}=\left[\mu^{+} 1_{\left[\omega \leq \omega^{*}\right]}-\mu^{-} 1_{\left[\omega>\omega^{*}\right]}\right] d t+\sigma_{Y} d W_{t}
$$

The stationary distribution of $\omega$,

$$
p(\omega)=\left\{\begin{array}{l}
p^{+}(\omega) \quad \omega \leq \omega^{*} \\
p^{-}(\omega) \quad \omega>\omega^{*}
\end{array}\right.
$$


exists if $0<\mu^{-}<\bar{i}$ and it satisfies the forward Kolmogorov ODE

$$
\frac{d^{2} p(\omega)}{d \omega^{2}}-\frac{2\left[\mu^{+} 1_{\left[\omega \leq \omega^{*}\right]}-\mu^{-} 1_{\left[\omega>\omega^{*}\right]}\right]}{\sigma_{Y}^{2}} \frac{d p(\omega)}{d \omega}=0
$$

$p(\omega)$ also satisfies the normalization condition

$$
\int_{-\infty}^{\omega^{*}} p^{+}(\omega) d \omega+\int_{\omega^{*}}^{\infty} p^{-}(\omega) d \omega=1
$$

Condition (B.4) eliminates a constant as a trivial solution of the ODE (B.3) which can be integrated once to yield

$$
\frac{d p(\omega)}{d \omega}-\frac{2\left[\mu^{+} 1_{\left[\omega \leq \omega^{*}\right]}-\mu^{-} 1_{\left[\omega>\omega^{*}\right]}\right]}{\sigma_{Y}^{2}} p(\omega)=0
$$

We can find a necessary boundary condition at $\omega=\omega^{*}$ by integrating equation (B.5) over the interval $\omega \in\left[\omega^{*}-\varepsilon, \omega^{*}+\varepsilon\right]$ and then taking a limit of $\varepsilon \rightarrow 0$ :

$$
\lim _{\varepsilon \rightarrow 0} \int_{\omega^{*}-\varepsilon}^{\omega^{*}+\varepsilon}\left[\frac{d p(\omega)}{d \omega}-\frac{2\left[\mu^{+} 1_{\left[\omega \leq \omega^{*}\right]}-\mu^{-} 1_{\left[\omega>\omega^{*}\right]}\right]}{\sigma_{Y}^{2}} p(\omega)\right] d \omega=p^{-}\left(\omega^{*}\right)-p^{+}\left(\omega^{*}\right)=0 .
$$

Therefore, we obtain that $p(\omega)$ is continuous at $\omega=\omega^{*}$. We now solve the ODE (B.3) separately for $p^{+}(\omega)$ and $p^{-}(\omega)$ :

$$
\begin{aligned}
& p^{+}(\omega)=A^{+} e^{\frac{2 \mu^{+}}{\sigma_{Y}^{2}} \omega}, \\
& p^{-}(\omega)=A^{-} e^{-\frac{2 \mu^{-}}{\sigma_{Y}^{2}} \omega},
\end{aligned}
$$

where $A^{ \pm}$can be found from the boundary and normalization conditions

$$
\begin{aligned}
& A^{+} e^{\frac{2 \mu^{+}}{\sigma_{Y}^{2}} \omega^{*}}=A^{-} e^{-\frac{2 \mu^{-}}{\sigma_{Y}^{2}} \omega^{*}}, \\
& \frac{\sigma_{Y}^{2}}{2 \mu^{+}} A^{+} e^{\frac{2 \mu^{+}}{\sigma_{Y}^{2}} \omega^{*}}+\frac{\sigma_{Y}^{2}}{2 \mu^{-}} A^{-} e^{-\frac{2 \mu^{-}}{\sigma_{Y}^{2}} \omega^{*}}=1 .
\end{aligned}
$$

Solving equations (B.8), (B.9) for $A^{+}$and $A^{-}$, we obtain (13). 


\section{Competitive equilibrium}

We did not establish rigorously that our proposed solution of the central planner's problem is correct. We have simply solved the HJB equation, without verifying that the resulting solution characterizes the value function. Since the central planner's problem is not a primary object of our interest, but rather an auxiliary device that we use to characterize a competitive equilibrium, such a proof is not necessary. Instead, we now prove that the investment policy derived as a solution of the central planner's problem is supported as an outcome of a competitive equilibrium.

PROPOSITION 1: A competitive equilibrium exists and the equilibrium investment policy is given by

$$
i_{t}^{*}=\left\{\begin{array}{l}
\bar{i}, \omega_{t} \leq \omega^{*} \\
0, \omega_{t}>\omega^{*}
\end{array}\right.
$$

where $\omega^{*}$ is given by (12).

Proof: We conjecture that the equilibrium investment policy $i_{t}^{*}$ is given by (C.10). Then, market clearing in the spot market implies that the spot price process is given by $S_{t}=e^{-\gamma \omega_{t}}$, where $\omega_{t}$ evolves according to (12).

A competitive firm chooses an investment policy $I_{t}$ to maximize the firm value, i.e., the present value of future output net of investment costs:

$$
\max _{I_{t}} \mathrm{E}_{0}\left[\int_{0}^{\infty} e^{-r t}\left(K_{t} e^{-\gamma \omega_{t}}-I_{t}\right) d t\right],
$$

subject to the capital accumulation rule

$$
\begin{gathered}
d K_{t}=\left(I_{t}-\delta K_{t}\right) d t \\
I_{t} \geq 0
\end{gathered}
$$




$$
I_{t} \leq \bar{\imath} K_{t}
$$

From (C.12), we obtain

$$
K_{t}=\int_{0}^{t} e^{-\delta(t-s)} I_{s} d s+K_{0} e^{-\delta t}
$$

Using this expression for the capital stock, and relaxing the constraint (C.14), we obtain a Lagrangian function for the above optimization problem:

$$
\begin{aligned}
\mathcal{L}= & \max _{I_{t} \geq 0} \mathrm{E}_{0}\left[\int_{0}^{\infty} e^{-r t}\left(\left[\int_{0}^{t} e^{-\delta(t-s)} I_{s} d s+K_{0} e^{-\delta t}\right] e^{-\gamma \omega_{t}}-I_{t}\right) d t+\right. \\
& \left.+\int_{0}^{\infty} e^{-r t} \lambda_{t}\left(\bar{\imath}\left[\int_{0}^{t} e^{-\delta(t-s)} I_{s} d s+K_{0} e^{-\delta t}\right]-I_{t}\right) d t\right] .
\end{aligned}
$$

Above, $e^{-r t} \lambda_{t} \geq 0$ is the Lagrange multiplier on the constraint on investment rate. Next, we change the order of integration in the integrals in (C.16) and obtain

$$
\mathcal{L}=\max _{I_{t} \geq 0} \mathrm{E}_{0}\left[\int_{0}^{\infty} e^{-r t}\left(V A_{t}+V I_{t}-1-\lambda_{t}\right) I_{t} d t\right]+K_{0}\left(V A_{0}+V I_{0}\right)
$$

where

$$
V A_{t}=\mathrm{E}_{t}\left[\int_{t}^{\infty} e^{-(r+\delta)(s-t)-\gamma \omega_{s}} d s\right]
$$

and

$$
V I_{t}=\bar{\imath}_{t}\left[\int_{t}^{\infty} e^{-(r+\delta)(s-t)} \lambda_{s} d s\right]
$$

Assuming that $V A_{t}$ and $V I_{t}$ are well defined (we will prove that below), the optimal solution of the relaxed problem is

$$
I_{t}^{*}=\left\{\begin{aligned}
\infty, & V A_{t}+V I_{t}-1-\lambda_{t}>0, \\
(0, \infty), & V A_{t}+V I_{t}-1-\lambda_{t}=0, \\
0, & V A_{t}+V I_{t}-1-\lambda_{t}<0 .
\end{aligned}\right.
$$

To obtain an optimal solution of the firm's original constrained problem, we need a particular choice of the Lagrange multiplier $\lambda_{t}$, for which the policy $I_{t}^{*}$ satisfies the original constraint, $I_{t}^{*} \leq \bar{i}\left(\int_{0}^{t} e^{-\delta(t-s)} I_{s} d s+K_{0} e^{-\delta t}\right)$, and the complimentary slackness condition, $\lambda_{t}\left(\bar{\imath}\left[\int_{0}^{t} e^{-\delta(t-s)} I_{s}^{*} d s+K_{0} e^{-\delta t}\right]-I_{t}^{*}\right)=0$. 
We recover the required Lagrange multiplier from the solution of the central planner's problem:

$$
\lambda_{t}=1_{\left[\omega_{t} \leq \omega^{*}\right]}\left(\frac{\partial J\left(K_{t}, Y_{t}\right)}{\partial K_{t}}-1\right)
$$

where $J\left(K_{t}, Y_{t}\right)$ is the value function of the central planner. Let $q_{t}=\partial J\left(K_{t}, Y_{t}\right) / \partial K_{t}\left(q_{t}\right.$ equals the Tobin's $\mathrm{Q}$ of firms in competitive equilibrium). The first-order condition on $J(K, Y)$ implies that $q_{t} \geq 1$ if and only if $\omega_{t} \leq \omega^{*}$. As we show below, our choice of $\lambda_{t}$ satisfies

$$
V A_{t}+V I_{t}=q_{t}
$$

We, therefore, find that if $\omega_{t} \leq \omega^{*}$, then $\lambda_{t}=q_{t}-1$ and therefore $V A_{t}+V I_{t}-1-\lambda_{t}=0$. Thus, in the region $\omega \leq \omega^{*}$, the optimal solution of the relaxed firm's problem can be chosen to satisfy the complimentary slackness condition, and thus it coincides with the conjectured equilibrium investment policy (C.10): $I_{t}^{*}=\bar{i}\left(\int_{0}^{t} e^{-\delta(t-s)} I_{s} d s+K_{0} e^{-\delta t}\right)$. In the region $\omega_{t}>\omega^{*}, \lambda_{t}=0$ and $V A_{t}+V I_{t}-1-\lambda_{t}<0$, hence $I_{t}^{*}=0$, which again coincides with the conjectured equilibrium policy (C.10).

To complete the proof, we need to establish an important technical result: we need to show that $V A_{t}+V I_{t}=q_{t}$, given that $\lambda_{t}=1_{\left[\omega_{t} \leq \omega^{*}\right]}\left(q_{t}-1\right)$. We proceed in two steps. Step 1. We first show that

$$
V A_{t}=\mathrm{E}_{t}\left[\int_{t}^{\infty} e^{-(r+\delta)(s-t)-\gamma \omega_{s}} d s\right]
$$

is finite and therefore

$$
\lim _{t \rightarrow \infty} \mathrm{E}_{0}\left[e^{-(r+\delta) t} e^{-\gamma \omega_{t}}\right]=0 .
$$

Let $B$ be an arbitrary positive number and define a stopping time $\tau_{B}=\inf \left\{t: \omega_{t} \leq\right.$ $\left.\omega^{*}-B\right\}$. Let

$$
F_{t}^{B}=\mathrm{E}_{t}\left[\int_{t}^{\infty} 1_{\left[s \leq \tau_{B}\right]} e^{-(r+\delta)(s-t)-\gamma \omega_{s}} d s\right] .
$$


We look for the answer in the form $F_{t}^{B}=F^{B}\left(\omega_{t}\right)$. We start by heuristically characterizing $F^{B}(\omega)$ as a unique solution of the Feynman-Kac equation

$$
\frac{\sigma_{Y}^{2}}{2} \frac{d^{2} F^{B}(\omega)}{d \omega^{2}}+\left[\bar{i} 1_{\left[\omega \leq \omega^{*}\right]}-\mu^{-}\right] \frac{d F^{B}(\omega)}{d \omega}-(r+\delta) F^{B}(\omega)+e^{-\gamma \omega}=0
$$

with the boundary condition

$$
F^{B}\left(\omega^{*}-B\right)=0
$$

We look for a solution of (C.23) of the form

$$
F^{B}(\omega)=\left\{\begin{array}{l}
A e^{\varkappa-\left(\omega-\omega^{*}\right)}+\frac{e^{-\gamma \omega}}{r-\gamma \mu^{-}-\frac{\gamma^{2}}{2} \sigma_{Y}^{2}}, \quad \omega \geq \omega^{*}, \\
C_{1} e^{\bar{\varkappa}_{+}\left(\omega-\omega^{*}\right)}+C_{2} e^{\bar{\varkappa}_{-}\left(\omega-\omega^{*}\right)}+\frac{e^{-\gamma \omega}}{r+\gamma \mu^{+}-\frac{\gamma^{2}}{2} \sigma_{Y}^{2}}, \quad \omega<\omega^{*} .
\end{array}\right.
$$

Substituting these solutions into ODE (C.23) yields quadratic equations on $\varkappa_{ \pm}$and $\bar{\varkappa}_{ \pm}$

$$
\begin{aligned}
& \frac{\sigma_{Y}^{2}}{2} \varkappa_{ \pm}^{2}+\mu^{-} \varkappa_{ \pm}-(r+\delta)=0 \\
& \frac{\sigma_{Y}^{2}}{2} \bar{\varkappa}_{ \pm}^{2}-\mu^{+} \bar{\varkappa}_{ \pm}-(r+\delta)=0 .
\end{aligned}
$$

$\varkappa_{ \pm}$and $\bar{\varkappa}_{ \pm}$denote, respectively, positive and negative roots of the above equations. Define

$$
M=\frac{\gamma \bar{\imath} e^{-\gamma \omega^{*}}}{\left(r+\delta-\gamma \mu^{-}-\frac{\gamma^{2}}{2} \sigma_{Y}^{2}\right)\left(r+\delta+\gamma \mu^{+}-\frac{\gamma^{2}}{2} \sigma_{Y}^{2}\right)} .
$$

Using the boundary condition (C.24) and imposing continuity of the function $F^{B}$ and its first derivative across $\omega^{*}$ (to verify that the solution of the differential equation (C.23) characterizes the expected value $F_{t}^{B}$, we only need the first derivative to be continuous at $\omega^{*}$ ), we obtain the following system of equations on coefficients $C_{1}, C_{2}$, and $A$ :

$$
\left\{\begin{array}{l}
C_{1}+C_{2}=A+M, \\
\bar{\varkappa}_{+} C_{1}+\bar{\varkappa}_{-} C_{2}=\left(\frac{2 \bar{\imath}}{\sigma_{Y}^{2}}+\varkappa_{-}\right) A+2 \frac{r+\delta+\gamma \mu^{+}-\gamma^{2} \sigma_{Y}^{2}}{\gamma \sigma_{Y}^{2}} M, \\
C_{1} e^{-\bar{\varkappa}_{+} B}+C_{2} e^{-\bar{\varkappa}_{-} B}=\frac{r+\delta-\gamma \mu^{-}-\frac{\gamma^{2}}{2} \sigma_{Y}^{2}}{\gamma \bar{\imath}} M e^{\gamma B} .
\end{array}\right.
$$

Solving this system yields the following expression for $C_{2}$ :

$$
C_{2}=M \frac{\frac{r+\delta-\gamma \mu^{-}-\frac{\gamma^{2}}{2} \sigma_{Y}^{2}}{\gamma \bar{\imath}}\left(\bar{\varkappa}_{+}-\frac{2 \bar{\imath}}{\sigma_{Y}^{2}}-\varkappa_{-}\right) e^{\left(\bar{\varkappa}_{-}+\gamma\right) B}-\left(2 \frac{r+\delta+\gamma \mu^{+}-\gamma^{2} \sigma_{Y}^{2}}{\gamma \sigma_{Y}^{2}}-\varkappa_{-}\right) e^{\left(\bar{\varkappa}_{-}-\bar{\varkappa}_{+}\right) B}}{\bar{\varkappa}_{+}-\frac{2 \bar{\imath}}{\sigma_{Y}^{2}}-\varkappa_{-}-\left(\bar{\varkappa}_{-}-\frac{2 \bar{\imath}}{\sigma_{Y}^{2}}-\varkappa_{-}\right) e^{\left(\bar{\varkappa}_{-}-\bar{\varkappa}_{+}\right) B}} .
$$


Next, we show that indeed $F_{t}^{B}=F^{B}\left(\omega_{t}\right)$. To see this, note that the process $X_{t}=$ $e^{-(r+\delta) t} F^{B}\left(\omega_{t}\right)+\int_{0}^{t} e^{-(r+\delta) s-\gamma \omega_{s}} d s$ is a local martingale. This follows from the fact that, by Ito's lemma, the drift of the process is equal to zero (due to (C.23)). Next, since the diffusion coefficient of $X_{t}, \sigma d F^{B}(\omega) / d \omega$, is bounded on the domain $\omega \geq \omega^{*}-B$, the stopped process $X_{t \wedge \tau_{B}}$ is a martingale. Thus,

$$
X_{0}=F^{B}\left(\omega_{0}\right)=\mathrm{E}_{0}\left[X_{T}\right]=\mathrm{E}_{0}\left[1_{\left[T \leq \tau_{B}\right]} e^{-(r+\delta) T} F^{B}\left(\omega_{T}\right)+\int_{0}^{T} 1_{\left[s \leq \tau_{B}\right]} e^{-(r+\delta) s-\gamma \omega_{s}} d s\right] .
$$

Since the function $F^{B}(\omega)$ is bounded on the domain $\left\{\omega_{t} \geq \omega^{*}-B\right\}$, we know that $\lim _{T \rightarrow \infty} \mathrm{E}_{0}\left[1_{\left[T \leq \tau_{B}\right]} e^{-(r+\delta) T} F^{B}\left(\omega_{T}\right)\right]=0$. Thus, as we take $T \rightarrow \infty$, by monotone convergence theorem, $F_{B}\left(\omega_{0}\right)=F_{0}^{B}$.

Having found $F_{t}^{B}$, we now take a limit of $B \rightarrow \infty$. The $\lim _{B \rightarrow \infty} F_{t}^{B}$ is well defined. Because $\frac{\sigma_{Y}^{2} \gamma^{2}}{2}+\gamma \mu^{+}-(r+\delta)<0$, it follows that $\bar{\varkappa}_{-}+\gamma<0$ and, therefore, $\lim _{B \rightarrow \infty} C_{2}=0$. Also, constants $C_{1}$ and $A$ converge to finite limits as $B \rightarrow \infty$.

To show that $\lim _{B \rightarrow \infty} F_{t}^{B}=V A_{t}$, we use the monotone convergence theorem, combined with an observation that $\lim _{B \rightarrow \infty} \tau_{B}=\infty$. The latter follows from the fact that $\omega_{t} \leq$ $\omega_{0}+\mu^{-} t+\sigma\left(W_{t}-W_{0}\right)$, which is an arithmetic Brownian motion and for which the corresponding stopping time converges to infinity as $B \rightarrow \infty$.

In summary, $V A_{t}$ is well defined, it is a function of the state variable, $V A\left(\omega_{t}\right)$, which satisfies the equation

$$
\frac{\sigma_{Y}^{2}}{2} \frac{d^{2} V A(\omega)}{d \omega^{2}}+\left[\bar{i} 1_{\left[\omega \leq \omega^{*}\right]}-\mu^{-}\right] \frac{d V A(\omega)}{d \omega}-(r+\delta) V A(\omega)+e^{-\gamma \omega}=0
$$

and is bounded from above by $A_{1}+A_{2} e^{-\gamma \omega}$ for a suitable choice of constants $A_{1}$ and $A_{2}$.

Step 2. We now prove that $V A_{t}+V I_{t}=q_{t}$. First, using the explicit solution of the central planner's problem, we find that $q$ satisfies

$$
1_{\left[\omega<\omega^{*}\right]} \bar{\imath}(q-1)+e^{-\gamma}-(r+\delta) q+\frac{\sigma_{Y}^{2}}{2} q_{\omega \omega}+\left(\bar{i} 1_{\left[\omega \leq \omega^{*}\right]}-\mu^{-}\right) q_{\omega}=0 .
$$


Next, define a process

$$
X_{t}=e^{-(r+\delta) t} q_{t}+\int_{0}^{t} e^{-(r+\delta) s}\left[e^{-\gamma \omega_{s}}+1_{\left[\omega_{s} \leq \omega^{*}\right]} \bar{i}\left(q_{s}-1\right)\right] d s .
$$

Given the above equation on $q(\omega)$, we see that $X_{t}$ is a local martingale. Using the explicit formula for $q(\omega)$, we also see that the diffusion coefficient of $X_{t}$ is bounded in absolute value by an affine function of $e^{-\gamma \omega}$. Since $\omega_{t}=\omega_{0}+\int_{0}^{t} i_{s}^{*} d s+\sigma\left(W_{t}-W_{0}\right)$ and $i_{s}^{*}$ is bounded, we conclude that the diffusion coefficient of $X_{t}$ has finite $L_{2}$ norm on compact time-intervals and therefore $X_{t}$ is a martingale. That, in turn, implies that

$$
X_{0}=q_{0}=\mathrm{E}_{0}\left[X_{T}\right]=\mathrm{E}_{0}\left[e^{-(r+\delta) T} q_{T}\right]+\mathrm{E}_{0}\left[\int_{0}^{T} e^{-(r+\delta) s}\left[e^{-\gamma \omega_{s}}+1_{\left[\omega_{s} \leq \omega^{*}\right]} \bar{i}\left(q_{s}-1\right)\right] d s\right]
$$

Since $q(\omega)$ is bounded in absolute value by an affine function of $e^{-\gamma \omega}$, we conclude that $\lim _{T \rightarrow \infty} \mathrm{E}_{0}\left[e^{-(r+\delta) T} q_{T}\right]=0$. This convergence result is a necessary condition for $V A_{t}$ to be finite and follows from Step 1 . Finally, since $1_{\left[\omega_{s} \leq \omega^{*}\right]} \bar{i}\left(q_{s}-1\right) \geq 0$, we can use the monotone convergence theorem to conclude that

$$
q_{0}=\lim _{T \rightarrow \infty} \mathrm{E}_{0}\left[\int_{0}^{T} e^{-(r+\delta) s}\left[e^{-\gamma \omega_{s}}+1_{\left[\omega_{s} \leq \omega^{*}\right]} \bar{i}\left(q_{s}-1\right)\right] d s\right]=V A_{0}+V I_{0} .
$$

This completes our proof. 


\section{Figure 1: Term structure of volatility, crude oil futures}

The data are daily percentage price changes on NYMEX crude oil (CL) futures from 1983 to $2000 . \sigma$ denotes the standard deviation of daily percentage price changes. The time to maturity is defined as number of months left until the delivery month. The unconditional standard deviation is constructed using sample's first and second moments, while standard deviations conditional on backwardation and contango are conditioned on the shape of the forward curve one day prior.

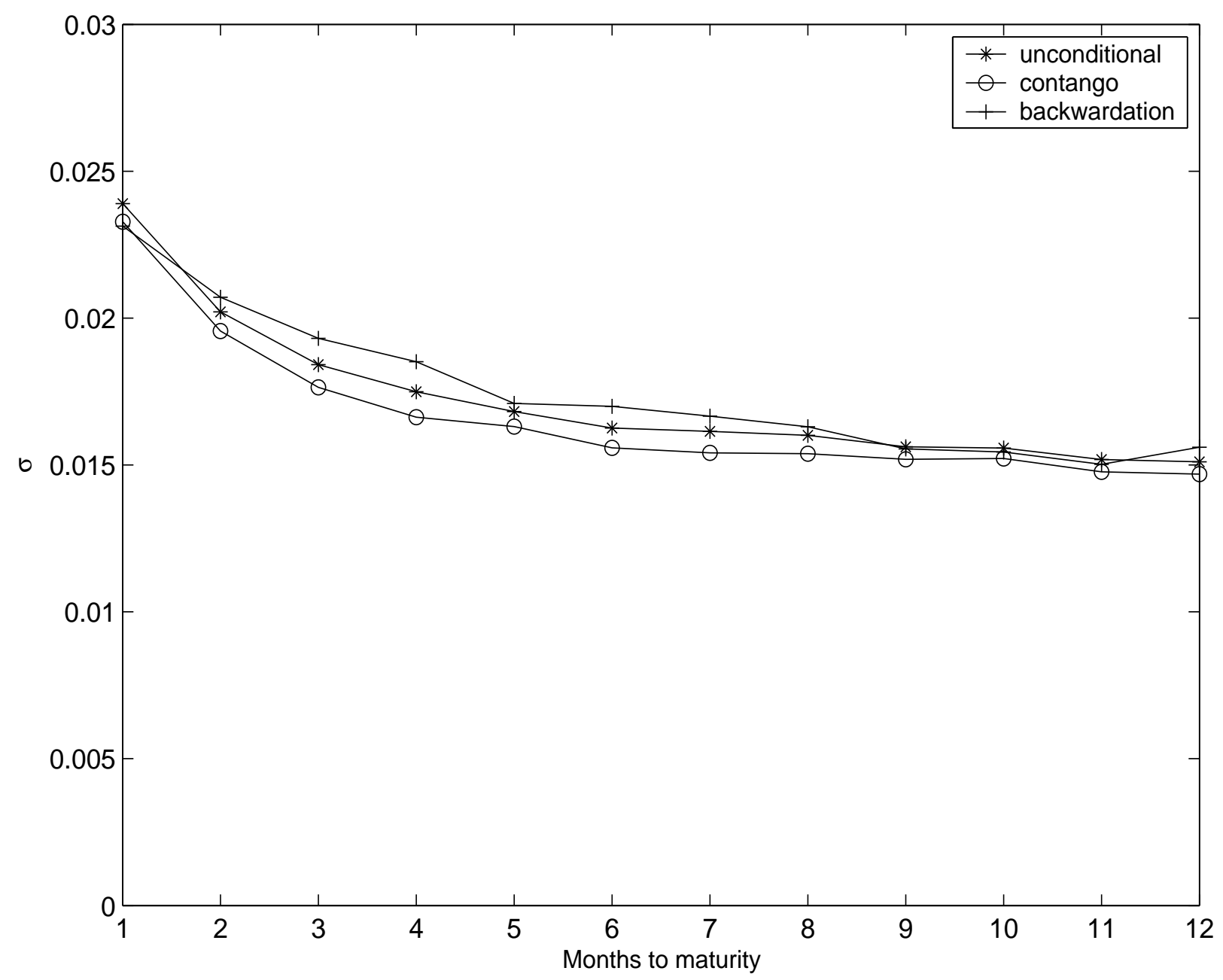


Figure 2: Term structure of volatility, heating oil futures

The data are daily percentage price changes on NYMEX heating oil (HO) futures from 1979 to 2000. See the caption to Figure 1 for details.

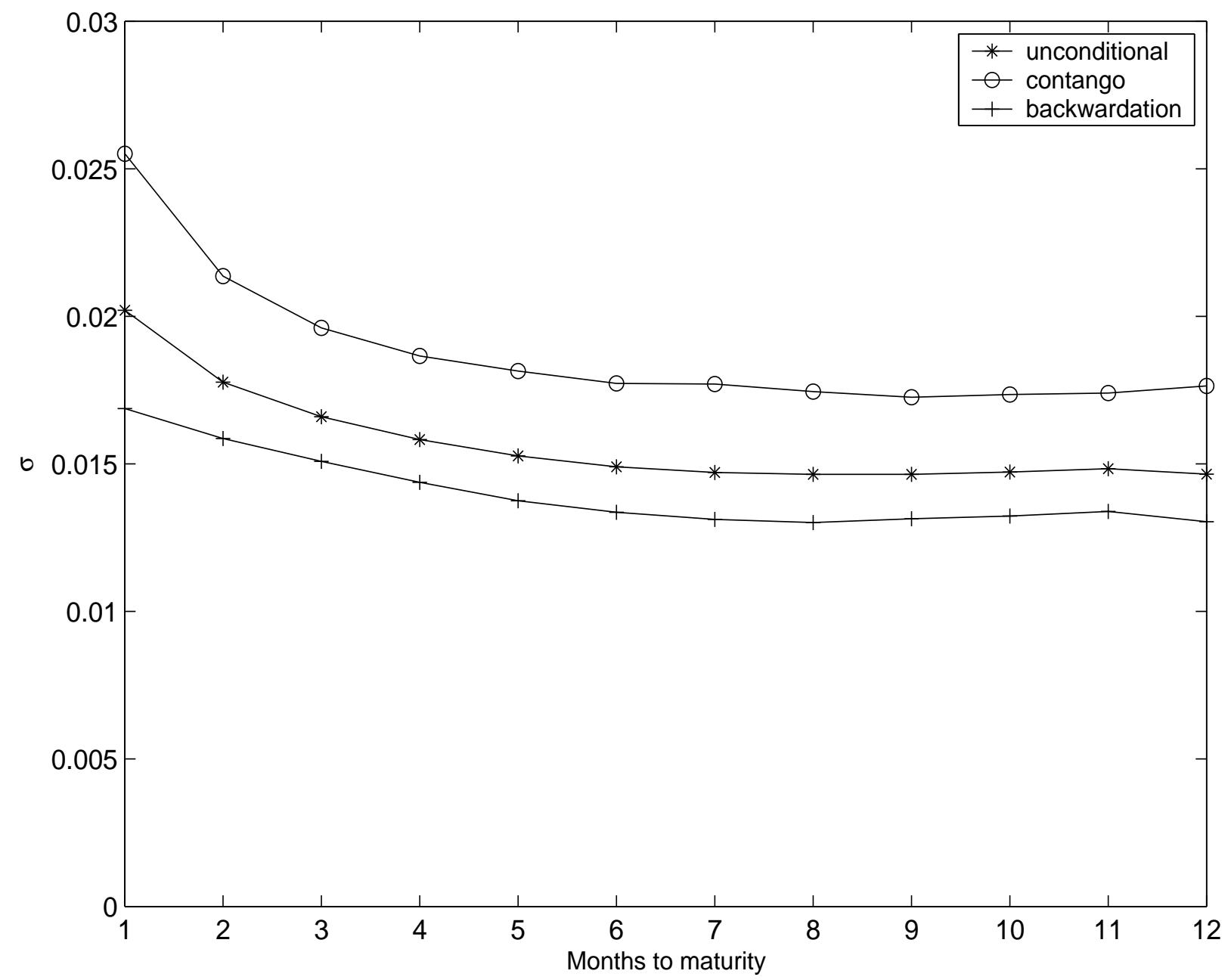


Figure 3: Term structure of volatility, unleaded gasoline futures

The data are daily percentage price changes on NYMEX unleaded gasoline (HU) futures from 1985 to 2000 . See the caption to Figure 1 for details.

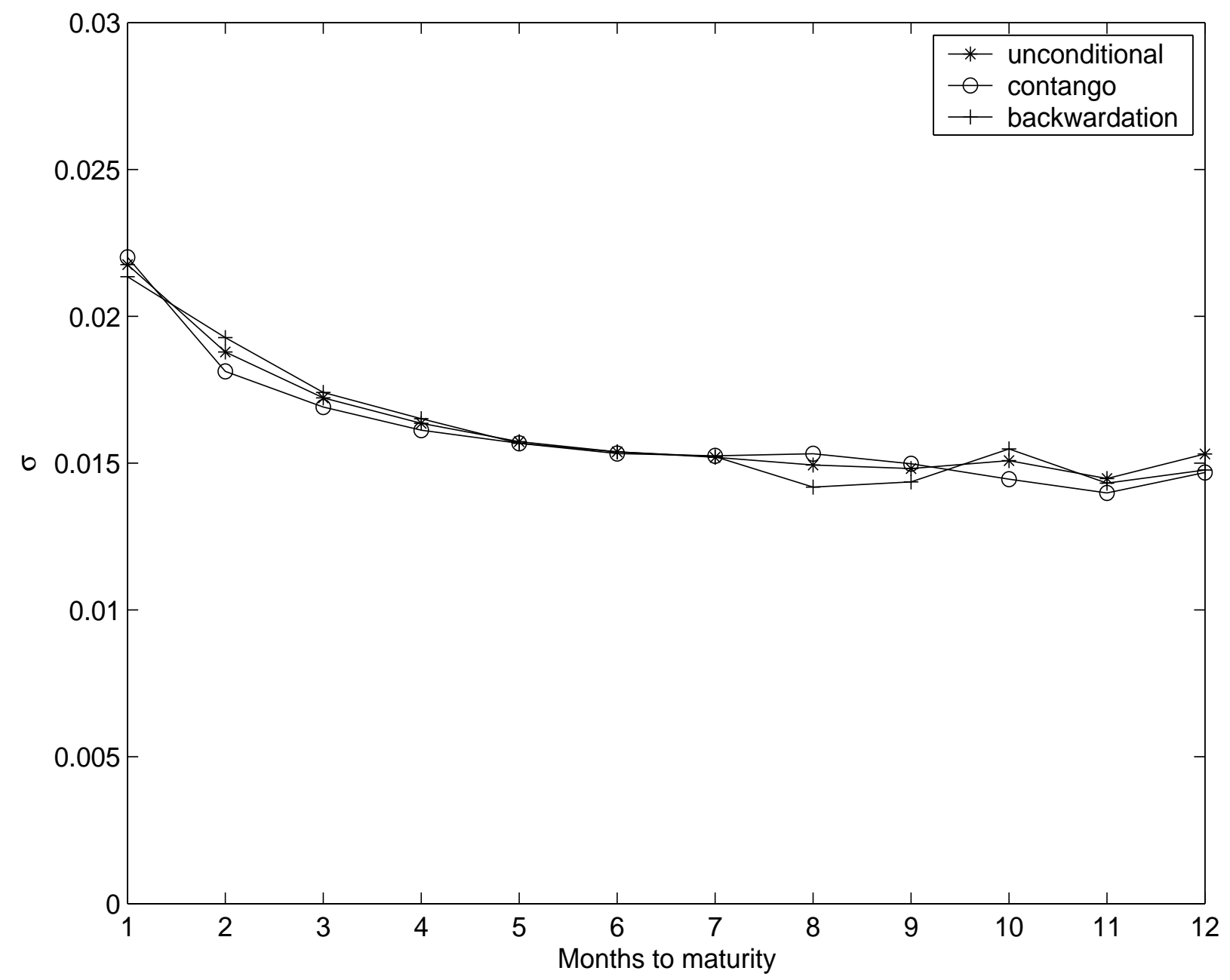




\section{Figure 4: Conditional volatility, crude oil futures}

The data are daily percentage price changes on NYMEX crude oil (CL) futures from 1983 to 2000, denoted by $R(t, T)$. Two different specifications are used to relate the instantaneous volatility of futures prices to the beginning-of-period slope of the forward curve. The first specification is

$$
|R(t, T)|=\alpha_{T}+\beta_{T} \ln \left[\frac{P(t-1,3)}{P(t-1,1)}\right]+\varepsilon_{T}(t) .
$$

The second specification decomposes the slope into positive and negative parts

$$
|R(t, T)|=\alpha_{T}+\beta_{1, T}\left(\ln \left[\frac{P(t-1,3)}{P(t-1,1)}\right]\right)^{+}+\beta_{2, T}\left(\ln \left[\frac{P(t-1,3)}{P(t-1,1)}\right]\right)^{-}+\varepsilon_{T}(t),
$$

where $(X)^{ \pm}$denotes positive (negative) part of $X$ respectively. The figure shows the estimates of all three coefficients (exhibit A) and their respective $t$-statistics (exhibit B) for different times to maturity. All $t$ statistics are White-adjusted.

(a) $\beta$

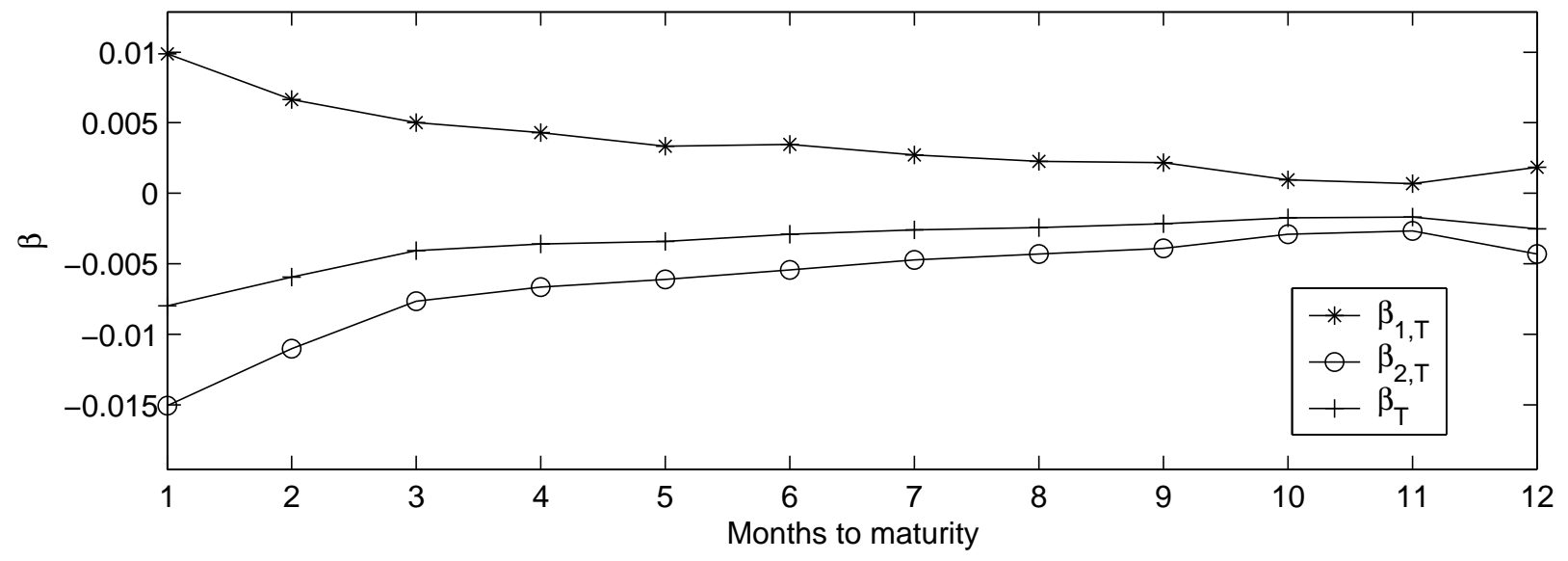

(b) $t$-statistics

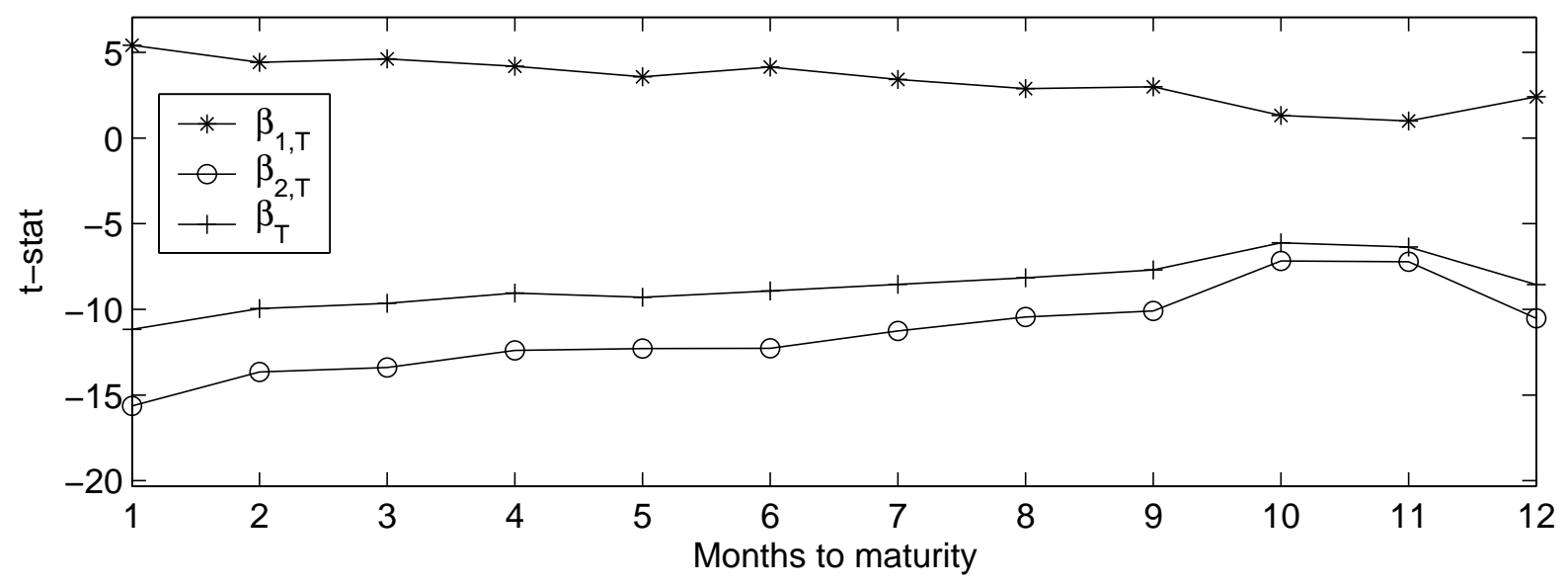


Figure 5: Conditional volatility, heating oil futures

The data are daily percentage price changes on NYMEX heating oil (HO) futures from 1979 to 2000. See the caption to Figure 4 for a detailed description.

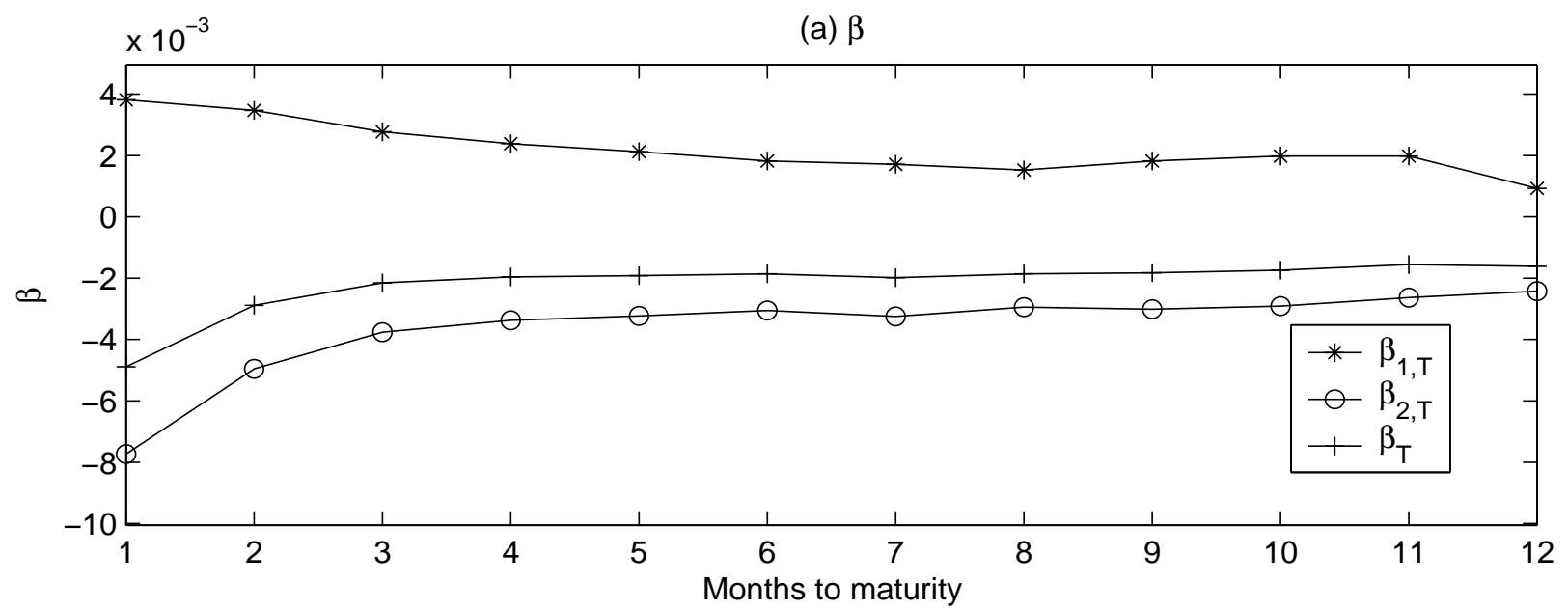

(b) $t$-statistics

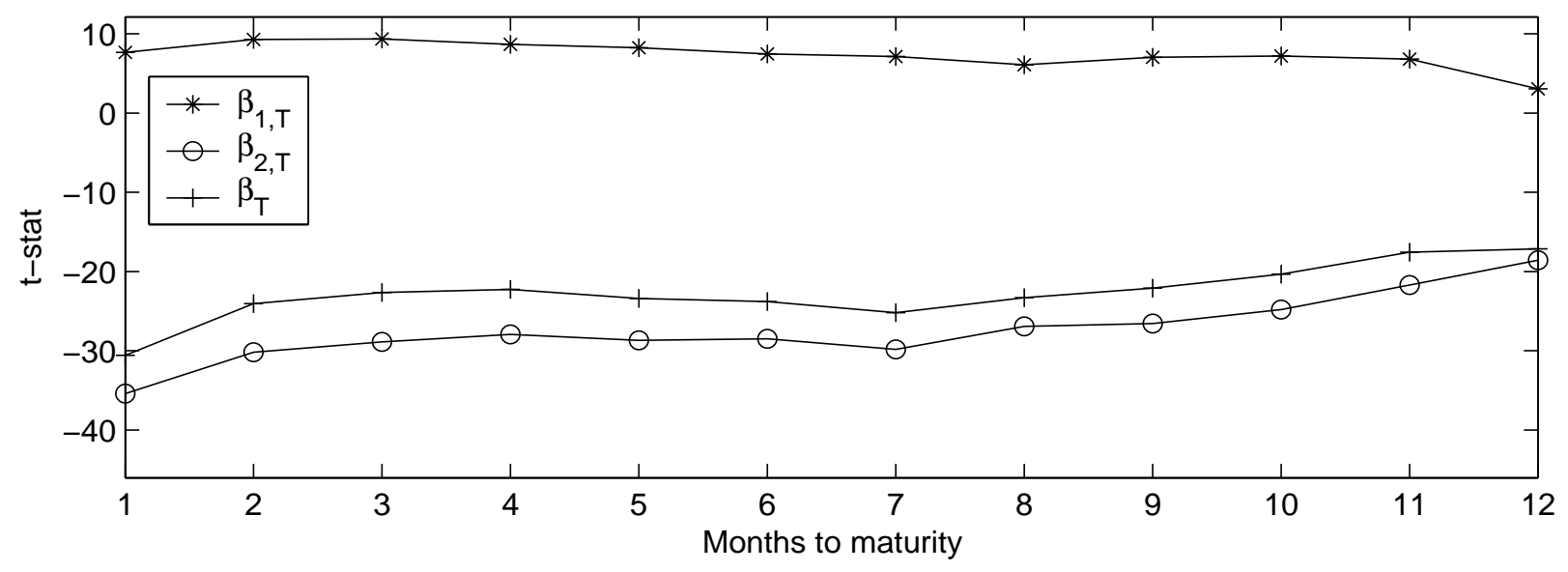


Figure 6: Conditional volatility, unleaded gasoline futures

The data are daily percentage price changes on NYMEX unleaded gasoline (HU) futures from 1985 to 2000. See the caption to Figure 4 for a detailed description.

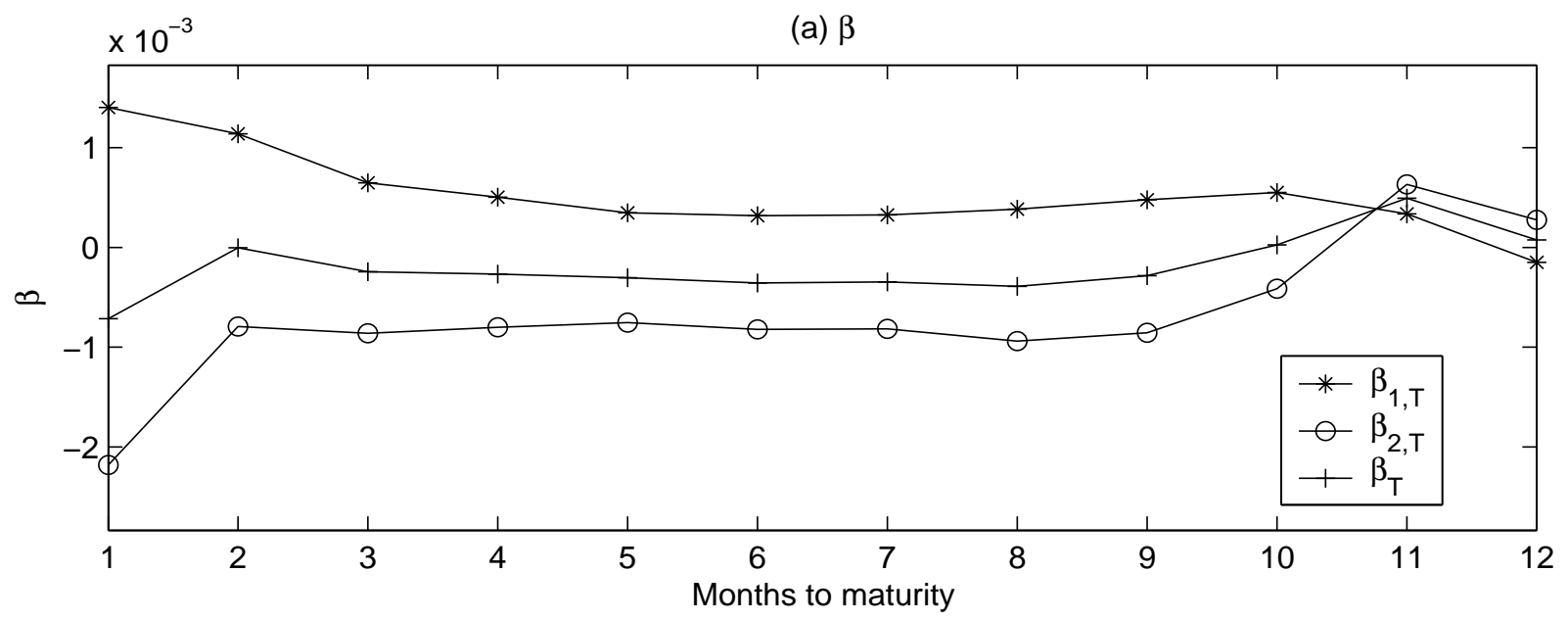

(b) $t$-statistics

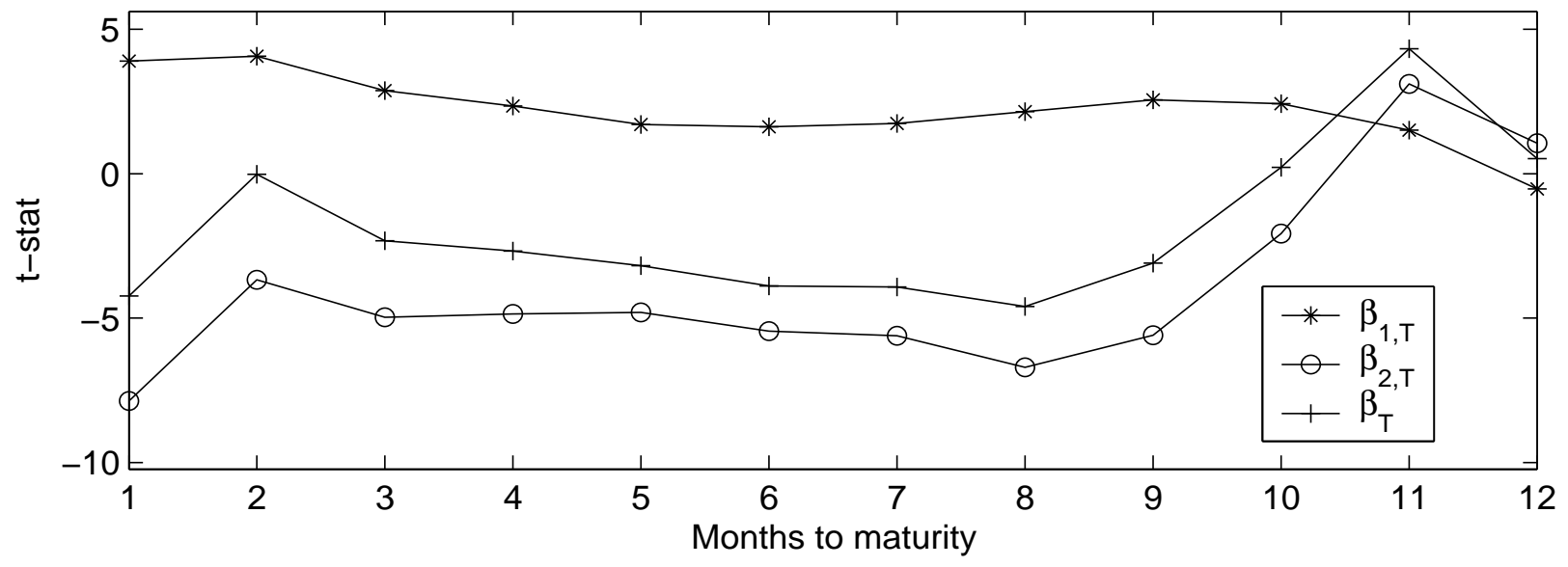




\section{Figure 7: Unconditional volatility of futures prices, model output}

The unconditional standard deviation of daily changes in futures prices is constructed based on the output from the model (model 1). The model is fitted to the data on crude oil futures (CL) using a two-step simulated method of moments described in Section 4. Parameter values are reported in Table 4 . Model 2 corresponds to the unconditional standard deviation of changes in futures prices implied by Eq. (20).

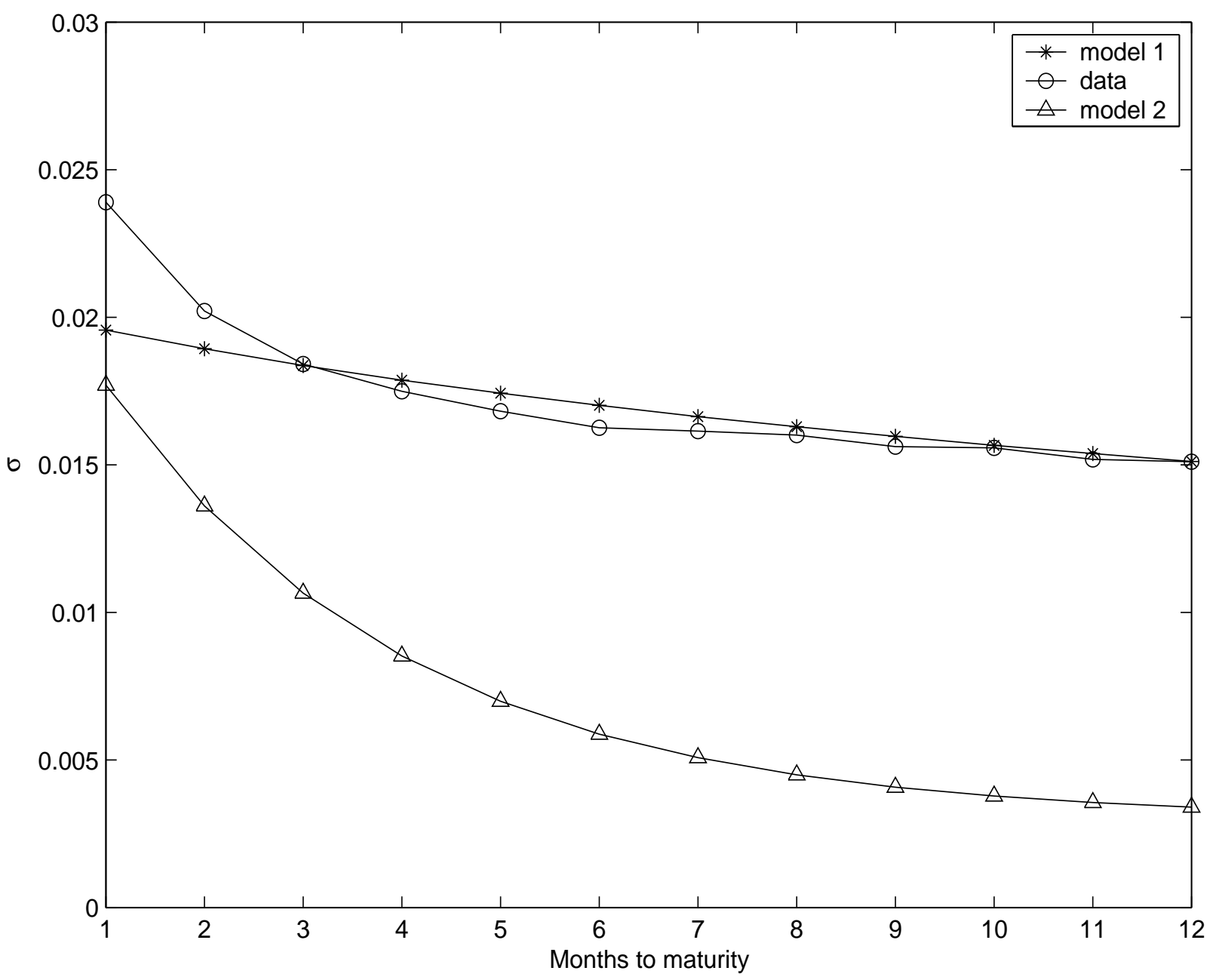




\section{Figure 8: Conditional volatility of futures prices, model output}

For each time to maturity, $T$, we simulate a time series of daily futures prices using parameter values reported in Table 4 . We then compute daily percentage changes in futures prices, denoted by $R(t, T)$. Two different specifications are used to relate the instantaneous volatility of futures prices to the beginning-of-period slope of the forward curve. The first specification is

$$
|R(t, T)|=\alpha_{T}+\beta_{T} \ln \left[\frac{P(t-1,3)}{P(t-1,1)}\right]+\varepsilon_{T}(t) .
$$

The second specification decomposes the slope into positive and negative parts

$$
|R(t, T)|=\alpha_{T}+\beta_{1, T}\left(\ln \left[\frac{P(t-1,3)}{P(t-1,1)}\right]\right)^{+}+\beta_{2, T}\left(\ln \left[\frac{P(t-1,3)}{P(t-1,1)}\right]\right)^{-}+\varepsilon_{T}(t),
$$

where $(X)^{ \pm}$denotes positive (negative) part of $X$ respectively. The figure shows all three betas for different times to maturity. See the caption to Figure 4 for further details.

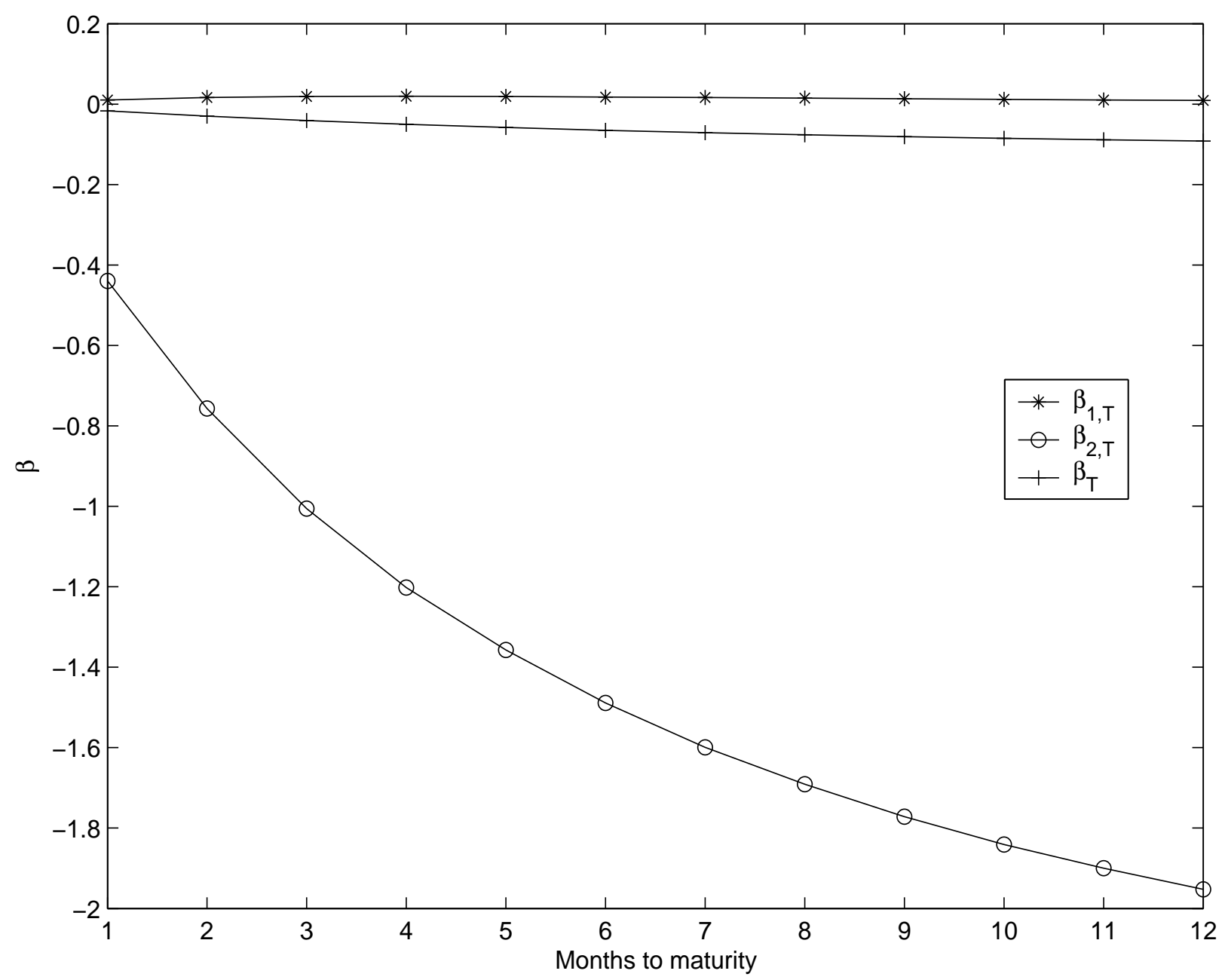


Figure 9: "V-shape" of volatility of futures prices, model output

Futures volatility is plotted as a function of the slope of the forward curve for different values of time to maturity. As a function of the slope, volatility exhibits a "V-shape" pattern. Volatility is expressed in annual terms. Model parameters are given in Table ??.

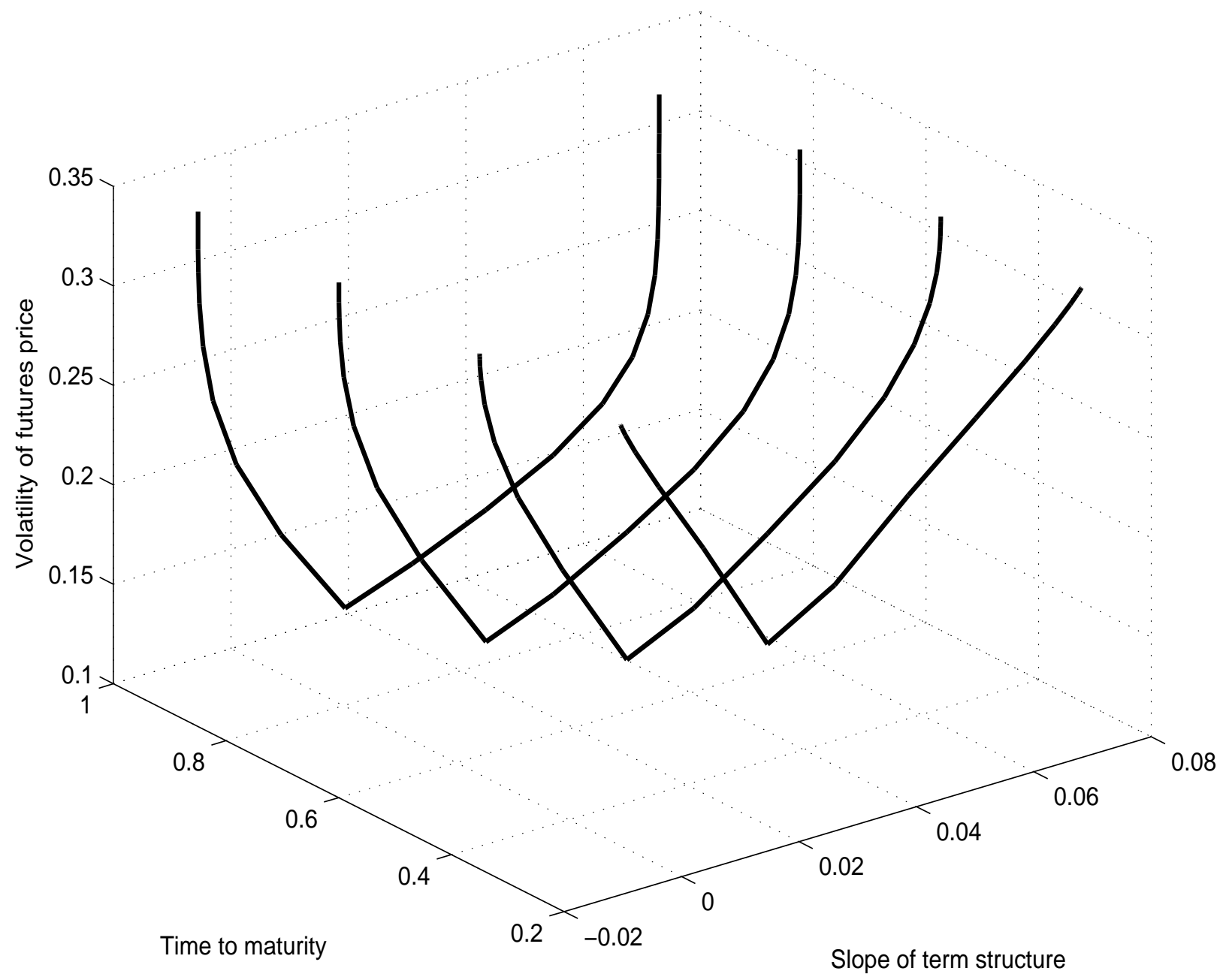




\section{Table 1: Conditional volatility, crude oil futures}

This Table reports results for three different regressions. The data are daily percentage price changes on NYMEX crude oil (CL) futures from 1983 to 2000. The specification in the both panels is the same as in Figure 4. All results are reported for times to maturity equal to one, five and ten months. All $t$-statistics are White-adjusted for conditional heteroscedasticity.

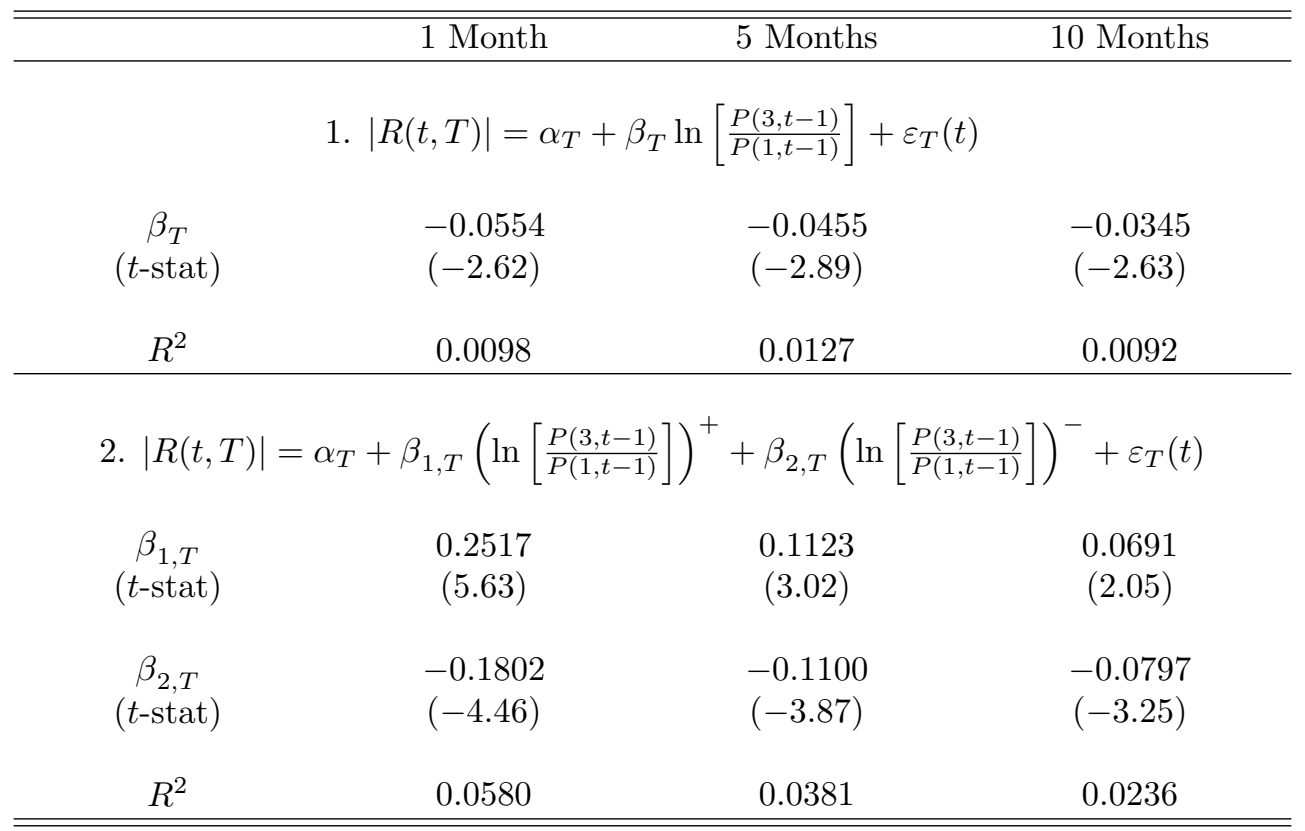




\section{Table 2: Conditional volatility, heating oil futures}

This table reports results for three different regressions. The data are daily percentage price changes on NYMEX heating oil (HO) futures from 1979 to 2000. See the caption to Table 1.

\begin{tabular}{|c|c|c|c|}
\hline & 1 Month & 5 Months & 10 Months \\
\hline \multicolumn{4}{|c|}{ 1. $|R(t, T)|=\alpha_{T}+\beta_{T} \ln \left[\frac{P(3, t-1)}{P(1, t-1)}\right]+\varepsilon_{T}(t)$} \\
\hline $\begin{array}{c}\beta_{T} \\
(t-\text { stat })\end{array}$ & $\begin{array}{l}-0.0711 \\
(-5.97)\end{array}$ & $\begin{array}{l}-0.0309 \\
(-3.51)\end{array}$ & $\begin{array}{l}-0.0359 \\
(-3.89)\end{array}$ \\
\hline$R^{2}$ & 0.0388 & 0.0140 & 0.0189 \\
\hline \multicolumn{4}{|c|}{ 2. $|R(t, T)|=\alpha_{T}+\beta_{1, T}\left(\ln \left[\frac{P(3, t-1)}{P(1, t-1)}\right]\right)^{+}+\beta_{2, T}\left(\ln \left[\frac{P(3, t-1)}{P(1, t-1)}\right]\right)$} \\
\hline $\begin{array}{c}\beta_{1, T} \\
(t-\text { stat })\end{array}$ & $\begin{array}{l}0.0523 \\
(4.54)\end{array}$ & $\begin{array}{c}0.0300 \\
(3.21)\end{array}$ & $\begin{array}{l}0.0192 \\
(2.14)\end{array}$ \\
\hline $\begin{array}{c}\beta_{2, T} \\
(t \text {-stat })\end{array}$ & $\begin{array}{l}-0.1450 \\
(-10.8)\end{array}$ & $\begin{array}{l}-0.0646 \\
(-4.65)\end{array}$ & $\begin{array}{l}-0.0632 \\
(-4.27)\end{array}$ \\
\hline$R^{2}$ & 0.0722 & 0.0473 & 0.0286 \\
\hline
\end{tabular}




\section{Table 3: Conditional volatility, unleaded gasoline futures}

This Table reports results for three different regressions. The data are daily percentage price changes on NYMEX unleaded gasoline (HU) futures from 1985 to 2000. See the caption to Table 1.

\begin{tabular}{|c|c|c|c|}
\hline & 1 Month & 5 Months & 10 Months \\
\hline \multicolumn{4}{|c|}{ 1. $|R(t, T)|=\alpha_{T}+\beta_{T} \ln \left[\frac{P(3, t-1)}{P(1, t-1)}\right]+\varepsilon_{T}(t)$} \\
\hline $\begin{array}{c}\beta_{T} \\
(t \text {-stat })\end{array}$ & $\begin{array}{l}-0.0337 \\
(-3.89)\end{array}$ & $\begin{array}{l}-0.0220 \\
(-3.43)\end{array}$ & $\begin{array}{l}-0.0222 \\
(-2.67)\end{array}$ \\
\hline$R^{2}$ & 0.0107 & 0.0084 & 0.0094 \\
\hline \multicolumn{4}{|c|}{ 2. $|R(t, T)|=\alpha_{T}+\beta_{1, T}\left(\ln \left[\frac{P(3, t-1)}{P(1, t-1)}\right]\right)^{+}+\beta_{2, T}\left(\ln \left[\frac{P(3, t-1)}{P(1, t-1)}\right]\right.$} \\
\hline $\begin{array}{c}\beta_{1, T} \\
(t \text {-stat })\end{array}$ & $\begin{array}{l}0.0442 \\
(3.45)\end{array}$ & $\begin{array}{c}0.0036 \\
(1.38)\end{array}$ & $\begin{array}{c}0.0009 \\
(1.09)\end{array}$ \\
\hline $\begin{array}{c}\beta_{2, T} \\
(t-\text { stat })\end{array}$ & $\begin{array}{l}-0.0795 \\
(-6.01)\end{array}$ & $\begin{array}{l}-0.0378 \\
(-3.37)\end{array}$ & $\begin{array}{l}-0.0476 \\
(-2.76)\end{array}$ \\
\hline$R^{2}$ & 0.0244 & 0.0114 & 0.0161 \\
\hline
\end{tabular}




\section{Table 4: Parameter estimates, crude oil futures}

This table reports our parameter values. We use a two step SMM procedure to estimate a vector of seven structural parameters $\theta \equiv\left\{\gamma, \mu_{Y}, \sigma_{Y}, \bar{i}, r, \delta, \lambda\right\}$. Since only five model parameters can be independently identified from the data (see Section 4), we fix $\delta$ and $r$ and estimate the remaining five parameters. We match the unconditional properties of futures prices, specifically the historic daily return on fully collateralized three-month futures position, the unconditional volatility of daily percent price changes for futures of various maturities as well as the mean, volatility, and the 30-day autoregressive coefficient of the slope of the forward curve. We use crude oil futures prices as a benchmark. In addition, we match the expected growth rate of crude oil consumption. The standard errors are reported where applicable.

\begin{tabular}{lcc}
\hline \hline Parameter & Value & Std. Error \\
\hline$\gamma$ & 3.4221 & 0.0396 \\
$\bar{i}$ & 0.1383 & 0.0031 \\
$r$ & 0.0200 & $\mathrm{NA}$ \\
$\mu_{Y}$ & 0.0115 & 0.0023 \\
$\sigma_{Y}$ & 0.0949 & 0.0116 \\
$\delta$ & 0.1200 & $\mathrm{NA}$ \\
$\lambda$ & $8.6 \times 10^{-6}$ & $8.4 \times 10^{-3}$ \\
\hline \hline
\end{tabular}




\section{Table 5: Conditional volatility, model output}

Conditional variance of crude oil futures prices are compared with the output of the model under two different econometric specifications. See the caption to Table 1.

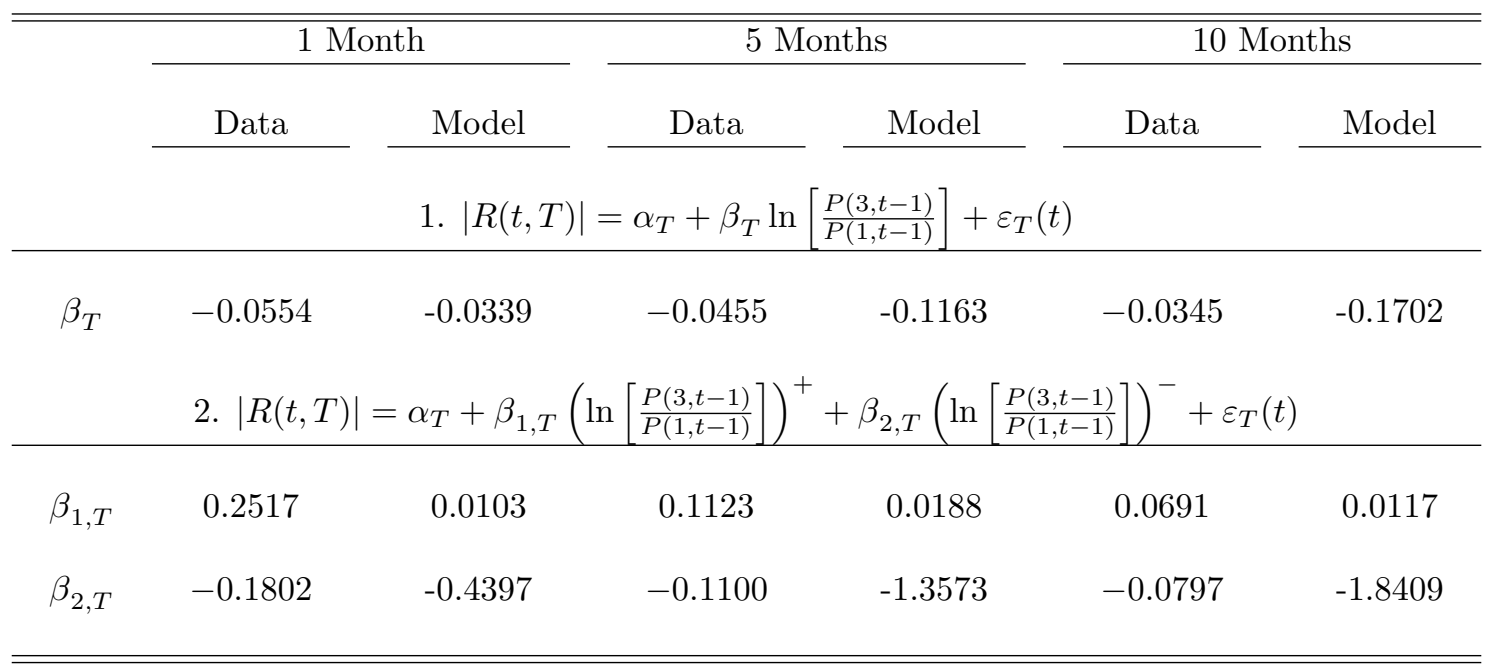




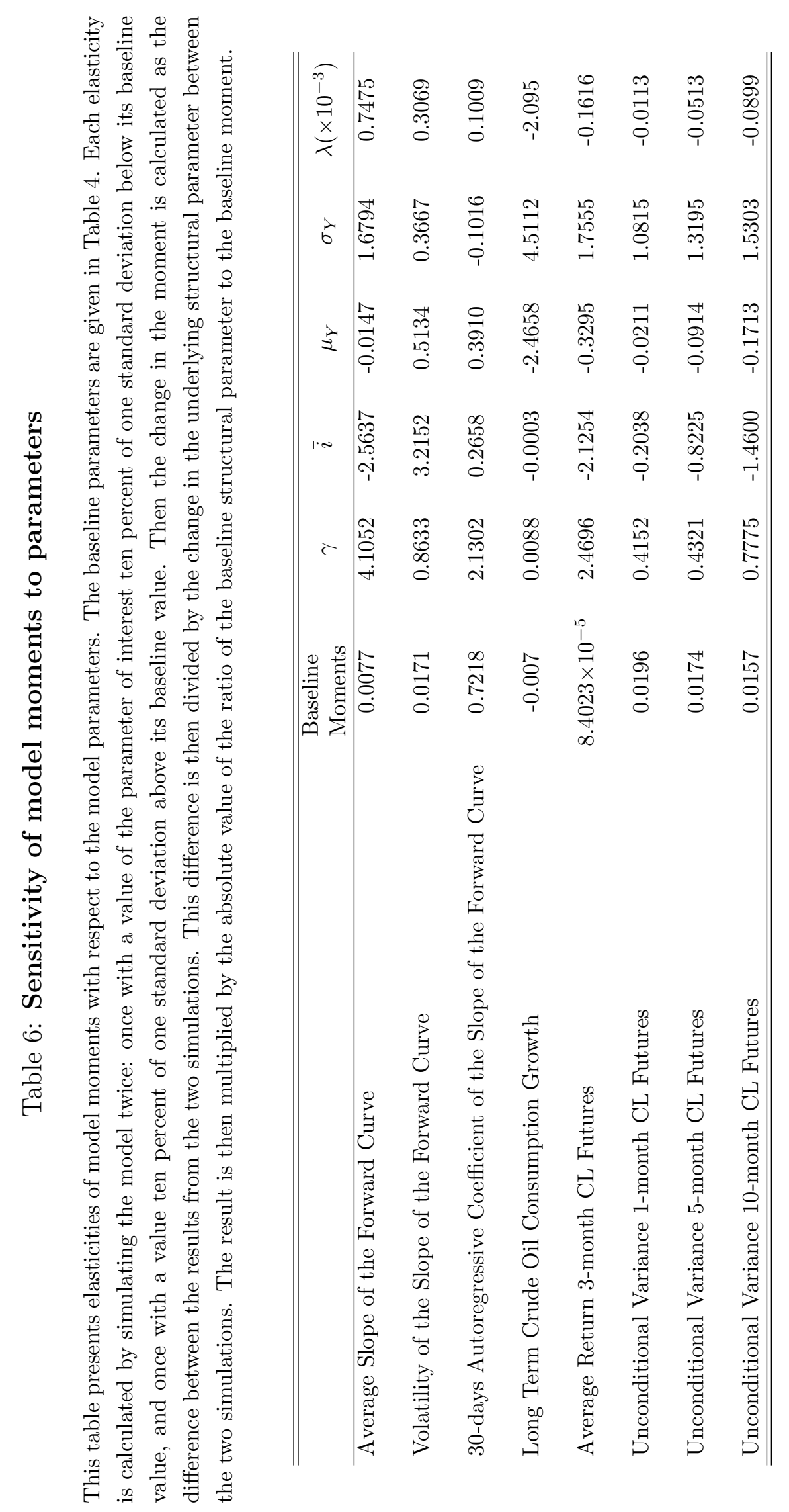

